\title{
The linear stability of double-diffusive miscible rectilinear displacements in a Hele-Shaw cell
}

\author{
David Pritchard ${ }^{\mathrm{a}, *}$ \\ ${ }^{a}$ Department of Mathematics, University of Strathclyde, 26 Richmond Street, \\ Glasgow G1 1XH, Scotland. Email:dtp@maths.strath.ac.uk.
}

\begin{abstract}
We investigate the viscous instability of a miscible displacement process in a rectilinear geometry, when the viscosity contrast is controlled by two quantities which diffuse at different rates. The analysis is applicable to displacement in a porous medium with two dissolved species, or to displacement in a Hele-Shaw cell with two dissolved species or with one dissolved species and a thermal contrast. We carry out asymptotic analyses of the linear stability behaviour in two regimes: that of small wavenumbers at intermediate times, and that of large times.

An interesting feature of the large-time results is the existence of regimes in which the favoured wavenumber scales with $t^{-1 / 4}$, as opposed to the $t^{-3 / 8}$ scaling found in other regimes including that of single-species fingering. We also show that the region of parameter space in which the displacement is unstable grows with time, and that although overdamped growing perturbations are possible, these are never the fastest-growing perturbations so are unlikely to be observed. We also interpret our results physically in terms of the stabilising and destabilising mechanisms acting on an incipient finger.
\end{abstract}

Key words: viscous fingering; instability.

\section{Introduction}

The instabilities which occur when a fluid of high mobility displaces a fluid of low mobility represent a classic fundamental problem in fluid mechanics with important practical applications (see, for example, the review by Homsy [1]).

* Corresponding author. Tel: +44(0)141 548 3819; fax: +44(0)141 5483345. 
Such instabilities occur under a variety of conditions, of which the simplest and most paradigmatic is flow in a homogeneous porous medium or the closely analogous system of a Hele-Shaw cell [2]. This problem is relevant to many industrial processes, in which instability and the subsequent fingering of one fluid into the other are typically undesirable effects, and considerable ingenuity has been devoted to eliminating them. In particular, efforts are often made to reduce the mobility of the displacing fluid below that of the fluid that it is displacing, by modifying either its temperature or its chemical composition. In nature, too, fingering instabilities may be relevant in controlling the mixing of fluids in porous rocks, and thus may affect the geochemical processes which take place and the consequent evolution of formations such as aquifers and oil reservoirs.

The earliest studies of viscous fingering (e.g. that by Saffman \& Taylor [2]) considered immiscible displacements, where the displacing and displaced fluids are separated by a sharp interface; in practice capillary forces often act on this interface and provide an important stabilising mechanism. Immiscible viscous fingering is relevant to the economically important process of enhanced oil recovery, in which oil is displaced from a porous medium by water that has been made more viscous by the addition of dissolved polymers [3,4]. In some other contexts, however, there is not a sharp interface between the displaced and displacing fluids. For example, in both freshwater aquifers and geothermal reservoirs, resident water may be displaced by injected or otherwise invading water carrying different dissolved species. (The viscosity of water can be significantly affected just by the concentration of common salts in solution. For example, seawater at a salinity of 35 and temperature of $25^{\circ}$ is around $9 \%$ more viscous than pure water at the same temperature: see [5], $\S \S 6-182$ and 14-15.) As the management of these resources becomes more important, it is likely that techniques developed in the oil industry will have to be applied, for example to extract fresh water rapidly from an aquifer or to drive incompatible invading water from a geothermal system before scaling can occur. Flows which are in some respects similar occur when warm (and thus less viscous) fluid displaces colder and more viscous fluid, for example as fresh magma invades a dyke. In all these contexts, the essential destabilising mechanisms leading to viscous fingering are the same as in immiscible fingering, but a key stabilising mechanism is the thermal or molecular diffusion of the fluid properties which control viscosity. The present study is therefore concerned with miscible displacements.

Most studies of miscible displacement have assumed that the viscosity contrast between the displaced and the displacing fluids is entirely controlled either by the presence of a single dissolved species or by the temperature of the fluid. However, in many contexts - for example, in the recharge of geothermal reservoirs [6] - the resident and injected fluids differ both in temperature and in composition: heat and solutes dissolve at different rates, and so the exchange 
of properties between them cannot be described in terms of a single diffusing quantity. Similar double-diffusive effects may occur when injected water is made more viscous by dissolving long-chain polymers in it (see e.g. [3,4]). Large polymer molecules have a substantially lower molecular diffusivity than other dissolved species (such as salts) which also affect the relative properties of the ambient and injectate, so in this situation too the effects of double diffusivity must be considered. The known complexity of gravitationally unstable displacements in double-diffusive and reactive-diffusive systems [7-9] suggests that these effects may not be simple to identify or to analyse. The object of the present study is to provide some insight into these effects by investigating the fundamental mechanisms of double-diffusive viscous fingering.

In a previous study [10], we investigated viscous fingering on a radially spreading displacement front in a porous medium, where both thermal and solutal components contributed to the viscosity contrast. In a porous medium, thermal signals are advected more slowly than the fluid because heat must be shared between the fluid and the porous matrix: the system is therefore 'double-advective' as well as double-diffusive [11], and spatially separated thermal and compositional fronts develop. The separation between these fronts controls how strongly they can stabilise or destabilise each other. By contrast, displacement processes in a well-insulated Hele-Shaw cell are 'iso-advective' and double-diffusive, and when the viscosity contrast depends on two differently diffusing dissolved species, the transport even in a porous medium becomes iso-advective. In this study, we therefore consider only iso-advective displacements: for simplicity, we phrase our discussion in terms of a displacement in a Hele-Shaw cell with a thermal and a compositional contrast, but we bear in mind that our results can be applied more generally.

A comment should be made about the validity of the Hele-Shaw flow model and its relevance as an analogue for porous media. It is key to the model that the spatial scale of all flow features is much greater than the gap width $\hat{b}$ of the Hele-Shaw cell, and that the Peclet number defined in terms of the gap width, the displacement velocity and the solutal concentration is not large. If these criteria are not satisfied then the flow becomes fully three-dimensional [12] and the gap-averaged equations defined below are no longer valid: the three-dimensional Stokes equations must be considered instead, leading to significantly different stability results [13]. Our analysis below will generally deal with relatively large length- and time-scales so this restriction is not crucial, but it should be borne in mind throughout.

A further difference between the present study and the earlier study by Pritchard [10] is that we consider a rectilinear rather than a radial displacement process. The radial geometry is particularly convenient from a mathematical perspective, since both the radius of the front and its streamwise width grow as $t^{1 / 2}$ : it is therefore possible to eliminate the time-dependency of the basic state 
by transforming to similarity variables (see e.g. [14]). In other geometries, this mathematical coincidence does not occur, and it is necessary to investigate the stability of a basic state which is evolving as the perturbation develops. (Note that this issue does not arise in some analogous problems, such as reactiondiffusion systems, where a steadily translating base state is available [8].) The standard approach is to 'freeze' time at some instant, and consider perturbations to the quasi-steady state at that instant (see e.g. [15]). However, Ben, Demekhin \& Chang [16] have recently argued that this approach is not justified because - especially at early times - the perturbations grow on timescales comparable to the timescale over which the basic state changes. Ben et al. developed a spectral method which could be used to obtain asymptotic results for the stability of long-wave perturbations, and we will adapt their method here. We consider rectilinear displacements, which are the simplest non-degenerate geometry available, and we believe that the insight obtained here may be more widely applicable.

This study is structured as follows. In $\S 2$ we formulate the governing equations. In $\S 3$ we carry out a linear stability analysis; we then develop asymptotic results for the growth rate of perturbations in two limits: that of long waves at intermediate times $(\S 4)$ and that of long times $(\S 5)$. Finally, in $\S 6$ we summarise our findings and their implications. Before proceeding, however, it is helpful to develop some simple hypotheses about the stability of the front, which will give us a baseline against which to compare our analysis.

\subsection{Some heuristic criteria for instability}

When double-diffusive viscous fingering occurs during a radial displacement, there are at least some cases in which a stabilising thermal gradient can stabilise an unstable concentration gradient [10]. It is not clear that this will be true for fingering of a rectilinear flow: the wavenumbers $m$ are not restricted in this case to be integers, so in principle, instability at any $m>0$ will be enough to trigger fingering. (It is generally found in stability analyses of viscous fingering that perturbations with very low wavenumbers are marginally unstable: this will be discussed further when we consider the spectral decomposition of perturbations in the streamwise direction.)

The most interesting situations occur when the temperature and compositional differences across the front contribute in different senses to the overall viscosity contrast, so one tends to stabilise and one to destabilise the front. The question is then whether the stabilising component can ever completely stabilise the front. Physical and heuristic reasoning supplies three arguments which predict substantially different results. 
(i) If solute and temperature diffuse at different rates, the fates of the two fields may become decoupled. This should mean that if either component promotes fingering then fingering will occur.

(ii) For the single-species problem, in the long-wave limit the growth rates are the same as in the immiscible case [16,1]. This suggests that for the two-species problem it should be the aggregate viscosity contrast which controls at least the marginal instability of the front.

(iii) It is also intuitively plausible that instability will occur if any part of the front is potentially unstable. In other words, if the global viscosity contrast across the front is stable but the viscosity profile is not monotonic, there will be a region within the front where less viscous fluid is displacing more viscous fluid, and instability may be expected. This agrees with some previous results $[17,18]$ for single-species fingering where the viscosity-concentration relation is non-monotonic.

We will return later to these arguments, and to the analogy with single-species fingering, in the light of our stability analysis.

\section{Governing equations}

We will present governing equations for flow in a Hele-Shaw cell with a temperature and a compositional contrast between the injected and ambient fluids: these equations are identical to those for the transport of two differently diffusing solutes in a porous medium, to within the limitations of the model as noted above.

We consider a Hele-Shaw cell with gap width $\hat{b}$; fluid is driven through it in the $\hat{x}$-direction at a constant displacement velocity $\hat{u}_{0}$. The continuity and Darcy equations are

$$
\hat{\nabla} \cdot \hat{\mathbf{u}}=0 \quad \text { and } \quad \hat{\mathbf{u}}=-\frac{\hat{K}}{\hat{\mu}} \hat{\nabla} \hat{p}
$$

where $\hat{K}=\hat{b}^{2} / 12$ is the effective permeability of the Hele-Shaw cell, and where the viscosity $\hat{\mu}$ depends both on the temperature $\hat{T}$ and the concentration $\hat{C}$ of some solute. The transport equations for $\hat{T}$ and $\hat{C}$ are

$$
\frac{\partial \hat{T}}{\partial \hat{t}}+(\hat{\mathbf{u}} \cdot \hat{\nabla}) \hat{T}=\hat{\kappa}_{T} \hat{\nabla}^{2} \hat{T} \quad \text { and } \quad \frac{\partial \hat{C}}{\partial \hat{t}}+(\hat{\mathbf{u}} \cdot \hat{\nabla}) \hat{C}=\hat{\kappa}_{C} \hat{\nabla}^{2} \hat{C} \text {. }
$$


The (idealised) boundary conditions can be expressed as

$$
\begin{gathered}
\hat{T} \rightarrow \hat{T}_{0}, \quad \hat{C} \rightarrow \hat{C}_{0} \quad \text { and } \quad \frac{\partial \hat{p}}{\partial \hat{x}} \rightarrow-\frac{\hat{\mu}_{0} \hat{u}_{0}}{\hat{K}} \quad \text { as } \quad \hat{x} \rightarrow-\infty ; \\
\hat{T} \rightarrow \hat{T}_{1}, \quad \hat{C} \rightarrow \hat{C}_{1} \quad \text { and } \quad \frac{\partial \hat{p}}{\partial \hat{x}} \rightarrow-\frac{\hat{\mu}_{1} \hat{u}_{0}}{\hat{K}} \quad \text { as } \quad \hat{x} \rightarrow \infty .
\end{gathered}
$$

(The subscript 0 denotes a downstream and the subscript 1 an upstream quantity.) Following previous studies from [15] onward, we take the viscosity to be given by

$$
\hat{\mu}=\hat{\mu}_{0} \exp \left[\hat{\beta}_{T}\left(\hat{T}-\hat{T}_{0}\right)+\hat{\beta}_{C}\left(\hat{C}-\hat{C}_{0}\right)\right] .
$$

To non-dimensionalise, we define

$$
\begin{gathered}
T=\frac{\hat{T}-\hat{T}_{0}}{\hat{T}_{1}-\hat{T}_{0}}, \quad C=\frac{\hat{C}-\hat{C}_{0}}{\hat{C}_{1}-\hat{C}_{0}}, \quad(x, y)=\left(\frac{\hat{x} \hat{u}_{0}}{\hat{\kappa}_{C}}, \frac{\hat{y} \hat{u}_{0}}{\hat{\kappa}_{C}}\right), \\
u=\frac{\hat{u}}{\hat{u}_{0}}, \quad t=\frac{\hat{t} \hat{u}_{0}^{2}}{\hat{\kappa}_{C}} \quad \text { and } \quad p=\frac{\hat{p} \hat{K}}{\hat{\mu}_{0} \hat{\kappa}_{C}} .
\end{gathered}
$$

The viscosity equation becomes

$$
\begin{aligned}
& \hat{\mu}=\hat{\mu}_{0} \mu(T, C), \quad \text { where } \quad \mu(T, C)=\mathrm{e}^{\beta_{T} T+\beta_{C} C} \\
& \text { and } \quad \beta_{T}=\hat{\beta}_{T}\left(\hat{T}_{1}-\hat{T}_{0}\right), \quad \beta_{C}=\hat{\beta}_{C}\left(\hat{C}_{1}-\hat{C}_{0}\right),
\end{aligned}
$$

and we note that the signs of $\beta_{C}$ and $\beta_{T}$ are not necessarily the same as those of $\hat{\beta}_{C}$ and $\hat{\beta}_{T}$.

The governing equations become

$$
\begin{gathered}
\nabla \cdot \mathbf{u}=0 \quad \text { and } \quad \mathbf{u}=-\frac{1}{\mu} \nabla p, \\
\frac{\partial T}{\partial t}+(\mathbf{u} . \nabla) T=L e \nabla^{2} T \quad \text { and } \quad \frac{\partial C}{\partial t}+(\mathbf{u} . \nabla) C=\nabla^{2} C,
\end{gathered}
$$

where the Lewis number $L e \equiv \kappa_{T} / \kappa_{c}$. If we take $T$ and $C$ to refer literally to heat and solute concentration, we expect that $L e \gg 1$; however, if we allow them to refer to concentrations of two dissolved species, we could have a much wider range of values. Without loss of generality, we will take 'temperature' to refer to the more rapidly diffusing field, and consider $L e>1$.

Finally, the boundary conditions become

$$
\begin{gathered}
T \rightarrow 0, \quad C \rightarrow 0 \quad \text { and } \quad \frac{\partial p}{\partial x} \rightarrow-1 \quad \text { as } \quad x \rightarrow-\infty ; \\
T \rightarrow 1, \quad C \rightarrow 1 \quad \text { and } \quad \frac{\partial p}{\partial x} \rightarrow-\mathrm{e}^{\beta_{T}+\beta_{C}} \quad \text { as } \quad x \rightarrow \infty .
\end{gathered}
$$




\section{$3 \quad$ Linear stability analysis}

We will seek perturbations to a basic state in which the displacement front is uniform in the $y$-direction, and widens diffusively in the $x$-direction. It is useful to define the moving co-ordinate $X=x-t$ and the self-similar variable $\xi=X / \sqrt{4 t}$; we may then write the basic state as

$$
C_{b}(X, t)=\frac{1}{2}+\frac{1}{2} \operatorname{erf}(\xi) \quad \text { and } \quad T_{b}(X, t)=\frac{1}{2}+\frac{1}{2} \operatorname{erf}\left(\frac{\xi}{\sqrt{L e}}\right) .
$$

The total viscosity contrast across the front is given by $\beta=\beta_{T}+\beta_{C}$. However, if $\operatorname{sign}\left(\beta_{T}\right) \neq \operatorname{sign}\left(\beta_{C}\right)$, the gradient may not have the same sign as $\beta$ everywhere. By symmetry, we know that the maximum 'contrary' gradient must occur at $\xi=0$. We have

$$
\left.\frac{\mathrm{d}\left(\log \mu_{b}\right)}{\mathrm{d} \xi}\right|_{\xi=0}=\frac{\beta_{C}+\beta_{T} / \sqrt{L e}}{\sqrt{\pi}},
$$

so the viscosity gradient will be non-monotonic if

$$
\left(\beta_{C}+\beta_{T}\right)\left(\beta_{C}+\frac{\beta_{T}}{\sqrt{L e}}\right)<0, \quad \text { i.e. } 1<-\frac{\beta_{T}}{\beta_{C}}<\sqrt{L e} .
$$

This is of interest because Manickam \& Homsy [18] found that instability occurred whenever the viscosity profile in their system was non-monotonic, although it sometimes occurred only after a finite 'waiting time'. We will compare this criterion with our results below.

We seek perturbations to the basic state, writing

$$
\begin{gathered}
u=1+\epsilon U_{1}(X, t) \mathrm{e}^{\mathrm{i} m y}, \quad v=\epsilon V_{1}(X, t) \mathrm{e}^{\mathrm{i} m y}, \quad p=p_{b}(X, t)+\epsilon P_{1}(X, t) \mathrm{e}^{\mathrm{i} m y}, \\
T=T_{b}(X, t)+\epsilon T_{1}(X, t) \mathrm{e}^{\mathrm{i} m y}, \quad \text { and } \quad C=C_{b}(X, t)+\epsilon C_{1}(X, t) \mathrm{e}^{\mathrm{i} m y}
\end{gathered}
$$

where $0<\epsilon \ll 1$. A little manipulation yields the perturbation equations

$$
\begin{aligned}
& \frac{\partial T_{1}}{\partial t}+U_{1} \frac{\partial T_{b}}{\partial X}=L e \frac{\partial^{2} T_{1}}{\partial X^{2}}-L_{e} m^{2} T_{1}, \quad \frac{\partial C_{1}}{\partial t}+U_{1} \frac{\partial C_{b}}{\partial X}=\frac{\partial^{2} C_{1}}{\partial X^{2}}-m^{2} C_{1} \\
& \text { and } \frac{\partial^{2} U_{1}}{\partial X^{2}}+\left(\beta_{T} \frac{\partial T_{b}}{\partial X}+\beta_{C} \frac{\partial C_{b}}{\partial X}\right) \frac{\partial U_{1}}{\partial X}-m^{2} U_{1}=m^{2}\left(\beta_{C} C_{1}+\beta_{T} T_{1}\right) .
\end{aligned}
$$

Substituting in the expressions

$$
\frac{\partial C_{b}}{\partial X}=\frac{1}{\sqrt{\pi}} \mathrm{e}^{-\xi^{2}} \frac{\partial \xi}{\partial X}=\frac{\mathrm{e}^{-\xi^{2}}}{2 \sqrt{\pi t}} \quad \text { and } \quad \frac{\partial T_{b}}{\partial X}=\frac{\mathrm{e}^{-\xi^{2} / L e}}{2 \sqrt{\pi L e t}}
$$


the perturbation equations become

$$
\begin{aligned}
& \frac{\partial T_{1}}{\partial t}+\frac{\mathrm{e}^{-\xi^{2} / L e}}{2 \sqrt{\pi L e t}} U_{1}=L e \frac{\partial^{2} T_{1}}{\partial X^{2}}-\operatorname{Le}^{2} T_{1}, \quad \frac{\partial C_{1}}{\partial t}+\frac{\mathrm{e}^{-\xi^{2}}}{2 \sqrt{\pi t}} U_{1}=\frac{\partial^{2} C_{1}}{\partial X^{2}}-m^{2} C_{1} \\
& \text { and } \frac{\partial^{2} U_{1}}{\partial X^{2}}+\left(\beta_{T} \frac{\mathrm{e}^{-\xi^{2} / L e}}{2 \sqrt{\pi L e t}}+\beta_{C} \frac{\mathrm{e}^{-\xi^{2}}}{2 \sqrt{\pi t}}\right) \frac{\partial U_{1}}{\partial X}-m^{2} U_{1}=m^{2}\left(\beta_{C} C_{1}+\beta_{T} T_{1}\right) .
\end{aligned}
$$

We now transform the independent variables to $(\xi, t)$, defining the rescaled perturbation quantities $\chi=2 \sqrt{\pi t} C_{1}$ and $\theta=2 \sqrt{\pi L e t} T_{1}$, to obtain

$$
\begin{gathered}
t \frac{\partial \theta}{\partial t}=\mathcal{L}_{T} \theta-m^{2} L e t \theta-\mathrm{e}^{-\xi^{2} / L e} t U_{1}, \quad \text { where } \quad \mathcal{L}_{T}=\left(\frac{1}{4} L e \frac{\partial^{2}}{\partial \xi^{2}}+\frac{1}{2} \xi \frac{\partial}{\partial \xi}+\frac{1}{2}\right), \\
t \frac{\partial \chi}{\partial t}=\mathcal{L}_{C} \theta-m^{2} t \theta-\mathrm{e}^{-\xi^{2}} t U_{1}, \quad \text { where } \quad \mathcal{L}_{C}=\left(\frac{1}{4} \frac{\partial^{2}}{\partial \xi^{2}}+\frac{1}{2} \xi \frac{\partial}{\partial \xi}+\frac{1}{2}\right), \quad(23) \\
\frac{\partial^{2} U_{1}}{\partial \xi^{2}}+\left(\beta_{T} \frac{\mathrm{e}^{-\xi^{2} / L e}}{\sqrt{\pi L e}}+\beta_{C} \frac{\mathrm{e}^{-\xi^{2}}}{\sqrt{\pi}}\right) \frac{\partial U_{1}}{\partial \xi}-4 t m^{2} U_{1}=\frac{2 \beta_{T} m^{2} \sqrt{t}}{\sqrt{\pi L e}} \theta+\frac{2 \beta_{C} m^{2} \sqrt{t}}{\sqrt{\pi}} \chi .
\end{gathered}
$$

The boundary conditions that the perturbations must satisfy are

$$
\theta \rightarrow 0, \quad \chi \rightarrow 0 \quad \text { and } \quad U_{1} \rightarrow 0 \quad \text { as } \quad \xi \rightarrow \pm \infty .
$$

\subsection{Spectral decomposition method}

Ben, Demekhin \& Chang [16] developed a spectral approach to miscible fingering when only a single diffusing species needs to be considered. The key to this method (see also [19], §8.3), is to consider the behaviour of perturbations in the long-wave limit $m=0$. In this limit, $U_{1}=0$ is the only solution satisfying the boundary conditions (25), and the evolution equations for $\theta$ and $\chi$ reduce to

$$
t \frac{\partial \theta}{\partial t}=\mathcal{L}_{T} \theta \quad \text { and } \quad t \frac{\partial \chi}{\partial t}=\mathcal{L}_{C} \theta
$$

with solutions which can be expressed in terms of the eigenfunctions of the operators $\mathcal{L}_{T}$ and $\mathcal{L}_{C}$ : these solutions essentially represent how a perturbation is damped by streamwise diffusion alone. The eigenfunctions of $\mathcal{L}_{C}$ are $\phi_{n}(\xi)=$ $H_{n}(\xi) \mathrm{e}^{-\xi^{2}}$ with eigenvalues $\sigma_{n}=-n / 2$ for $n=0,1,2 \ldots$, where $H_{n}$ are the Hermite polynomials. The corresponding eigenfunctions of $\mathcal{L}_{T}$ are therefore $\psi_{n}=H_{n}(\xi / \sqrt{L e}) \mathrm{e}^{-\xi^{2} / L e}$ with the same eigenvalues. 
The crucial point is that the operators $\mathcal{L}_{T}$ and $\mathcal{L}_{C}$ have discrete eigenspectra, and the only non-negative eigenvalue in each case is zero, so the only streamwise mode which does not decay under streamwise diffusion is the zero eigenfunction. (It can be shown that this zero eigenfunction corresponds to an infinitesimal change in the spatial origin of the basic solution: see [19], §8.3.) Therefore, at least for small positive values of the wavenumber $m$, we expect that the streamwise structure of the perturbation will continue to be dominated by the zero modes $\phi_{0}(\xi)$ and $\psi_{0}(\xi)$, at least after an initial decay time of order 1 . Hence to investigate possible instabilities of the system it should be sufficient to consider only the projection of the perturbation equations (22)(24) onto these dominant modes. We may carry out such a projection using the inner products

$$
\langle f(\xi)\rangle_{C}=\frac{1}{\sqrt{\pi}} \int_{-\infty}^{\infty} f(\xi) \mathrm{d} \xi \quad \text { and } \quad\langle f(\xi)\rangle_{T}=\frac{1}{\sqrt{\pi L e}} \int_{-\infty}^{\infty} f(\xi) \mathrm{d} \xi,
$$

so that $\left\langle\psi_{n}\right\rangle_{T}=\delta_{0 n}=\left\langle\phi_{n}\right\rangle_{C}$.

Substituting in the approximations $\chi \sim A(t) \phi_{0}(\xi)$ and $\theta \sim B(t) \psi_{0}(\xi)$ and taking the appropriate inner products, we obtain

$$
\frac{\mathrm{d} A}{\mathrm{~d} t}=-m^{2} A-\left\langle\mathrm{e}^{-\xi^{2}} U_{1}\right\rangle_{C} \quad \text { and } \quad \frac{\mathrm{d} B}{\mathrm{~d} t}=-m^{2} L e B-\left\langle\mathrm{e}^{-\xi^{2} / L e} U_{1}\right\rangle_{T},
$$

while the equation which governs $U_{1}$ becomes

$$
\begin{aligned}
\frac{\partial^{2} U_{1}}{\partial \xi^{2}}+\left(\beta_{T} \frac{\mathrm{e}^{-\xi^{2} / L e}}{\sqrt{\pi L e}}+\beta_{C} \frac{\mathrm{e}^{-\xi^{2}}}{\sqrt{\pi}}\right) \frac{\partial U_{1}}{\partial \xi}-4 t m^{2} U_{1} & = \\
& \frac{2 \beta_{C} m^{2} \sqrt{t}}{\sqrt{\pi}} A(t) \mathrm{e}^{-\xi^{2}}+\frac{2 \beta_{T} m^{2} \sqrt{t}}{\sqrt{\pi L e}} B(t) \mathrm{e}^{-\xi^{2} / L e}
\end{aligned}
$$

The explicit dependence of equation (29) on $t$, as well as the $\xi$-dependence of the left-hand side, means that we are unable to obtain an explicit solution to the perturbation problem in general. Following [16] we can, however, obtain asymptotic solutions which are valid, effectively, at intermediate and at late times, and from them we can obtain some insight into the general behaviour of the system.

\section{Long-wave, intermediate-time asymptotics}

Since the growth rate of perturbations reduces to zero at zero wavenumber and must be negative for very large wavenumbers due to transverse diffusion, it is natural to examine the regime $0<m \ll 1$ for evidence of (marginal) 
instability. In the following analysis we will do this while considering times $t=\mathcal{O}(1)$; this latter criterion ensures that higher streamwise modes present in the initial condition have decayed, leaving the perturbation dominated by $\phi_{0}(\xi)$ and $\psi_{0}(\xi)$ as required by $(29)$.

Following [16], we also assume that $\left|\beta_{T}\right|$ and $\left|\beta_{C}\right|$ are sufficiently small that we may neglect the terms proportional to them on the left hand side of (29). Appendix A demonstrates that this is legitimate, and that for small viscosity contrasts the corrections are of order $\left(\left|\beta_{C}\right|+\left|\beta_{T}\right|\right)^{3}$. We then obtain

$$
\frac{\partial^{2} U_{1}}{\partial \xi^{2}}-4 t m^{2} U_{1} \sim \frac{2 \beta_{C} m^{2} \sqrt{t}}{\sqrt{\pi}} A(t) \mathrm{e}^{-\xi^{2}}+\frac{2 \beta_{T} m^{2} \sqrt{t}}{\sqrt{\pi L e}} B(t) \mathrm{e}^{-\xi^{2} / L e}
$$

with solution

$$
\begin{gathered}
U_{1}(\xi, t)=\frac{1}{4} m \mathrm{e}^{2 m \sqrt{t} \xi}\left[\beta_{C} A(t) \mathrm{e}^{m^{2} t}(\operatorname{erf}(\xi+m \sqrt{t})-1)\right. \\
\left.+\beta_{T} B(t) \mathrm{e}^{m^{2} L e t}\left(\operatorname{erf}\left(\frac{\xi}{\sqrt{L e}}+m \sqrt{L e t}\right)-1\right)\right] \\
-\frac{1}{4} m \mathrm{e}^{-2 m \sqrt{t} \xi}\left[\beta_{C} A(t) \mathrm{e}^{m^{2} t}(\operatorname{erf}(\xi-m \sqrt{t})+1)\right. \\
\left.+\beta_{T} B(t) \mathrm{e}^{m^{2} \operatorname{Let}}\left(\operatorname{erf}\left(\frac{\xi}{\sqrt{L e}}-m \sqrt{L e t}\right)+1\right)\right]+\mathcal{O}\left(\left(\left|\beta_{C}\right|+\left|\beta_{T}\right|\right)^{3}\right) .
\end{gathered}
$$

Expanding this for $m \ll 1$, we have

$$
\begin{aligned}
U_{1}(\xi, t) \sim-\frac{1}{2} m\left(\beta_{C} A(t)\right. & \left.+\beta_{T} B(t)\right)+\frac{m^{2}}{\sqrt{\pi}}\left[A(t) \beta_{C}\left(\mathrm{e}^{-\xi^{2}} \sqrt{t}+\sqrt{\pi t} \xi \operatorname{erf}(\xi)\right)\right. \\
+ & \left.B(t) \beta_{T}\left(\mathrm{e}^{-\xi^{2} / L e} \sqrt{L e t}+\sqrt{\pi t} \xi \operatorname{erf}\left(\frac{\xi}{\sqrt{L e}}\right)\right)\right]
\end{aligned}
$$

We may therefore evaluate the inner products approximately as

$$
\left\langle U_{1} \phi_{0}\right\rangle_{C} \sim\left(-\frac{\beta_{C}}{2} m+\sqrt{\frac{2 t}{\pi}} \beta_{C} m^{2}\right) A(t)+\left(-\frac{\beta_{T}}{2} m+\beta_{T} \sqrt{t} \sqrt{\frac{1+L e}{\pi}} m^{2}\right) B(t)
$$

and

$$
\left\langle U_{1} \psi_{0}\right\rangle_{T} \sim\left(\beta_{C} \sqrt{t} \sqrt{\frac{1+L e}{\pi}} m^{2}-\frac{\beta_{C}}{2} m\right) A(t)+\left(\sqrt{\frac{2 L e t}{\pi}} \beta_{T} m^{2}-\frac{\beta_{T}}{2} m\right) B(t) .
$$


Substituting these into equations (28), we obtain the amplitude evolution equations

$$
\frac{\mathrm{d}}{\mathrm{d} t}\left(\begin{array}{l}
A \\
B
\end{array}\right)=\left(\begin{array}{ll}
E_{11} & E_{12} \\
E_{21} & E_{22}
\end{array}\right) \cdot\left(\begin{array}{l}
A \\
B
\end{array}\right)
$$

where the components of the matrix $\mathrm{E}$ are given by

$$
\begin{gathered}
E_{11}=-m^{2}+\frac{\beta_{C}}{2} m-\sqrt{\frac{2 t}{\pi}} \beta_{C} m^{2}, \quad E_{12}=\frac{\beta_{T}}{2} m-\beta_{T} \sqrt{t} \sqrt{\frac{1+L e}{\pi}} m^{2}, \\
E_{21}=\frac{\beta_{C}}{2} m-\beta_{C} \sqrt{t} \sqrt{\frac{1+L e}{\pi}} m^{2}, \quad E_{22}=-m^{2} L e+\frac{\beta_{T}}{2} m-\sqrt{\frac{2 L e t}{\pi}} \beta_{T} m^{2} .
\end{gathered}
$$

The eigenvalues of $\mathrm{E}$, and thus the growth rates of perturbations, are given by

$$
\sigma_{ \pm}=\frac{1}{2}\left(E_{11}+E_{22}\right) \pm \frac{1}{2}\left[\left(E_{11}-E_{22}\right)^{2}+4 E_{12} E_{21}\right]^{1 / 2} .
$$

The structure of the corresponding perturbations is given by the ratio of the solutal to the thermal perturbation amplitude,

$$
\left(\frac{B}{A}\right)_{ \pm}=\frac{E_{22}-E_{11} \pm \sqrt{\left(E_{11}-E_{22}\right)^{2}+4 E_{12} E_{21}}}{2 E_{12}} .
$$

\subsection{Reduction to the single-species case}

For the particular case $\beta_{T}=0$, the problem reduces to that of single-species miscible fingering, as considered by [16]. The predicted asymptotic growth rate at early times is then equal to $E_{11}$. This has the same form as the growth rate predicted by Ben et al.'s analysis (their equation (45)), but it differs by a factor of $\sqrt{\pi}$ in the final term. This appears to be due to an algebraic error in equation (33) of the earlier paper, where a spurious factor of $\mathrm{e}^{-\xi^{2}}$ is introduced.

It is interesting to note that in dimensional terms, the growth rate of perturbations for disturbances of long wavelength scales as

$$
\hat{\sigma} \sim \frac{1}{2} \hat{m} \hat{u}_{0} \log \left(\hat{\mu}_{1} / \hat{\mu}_{0}\right)=\hat{m} \hat{u}_{0} \frac{\hat{\mu}_{1}-\hat{\mu}_{0}}{\hat{\mu}_{1}+\hat{\mu}_{0}}+\mathcal{O}\left(\left(\frac{\hat{\mu}_{1}-\hat{\mu}_{0}}{\hat{\mu}_{0}}\right)^{2}\right),
$$

so for small viscosity contrasts the predicted growth rate is identical to the classic result of Saffman \& Taylor [2] for immiscible fingering. Ben et al. describe this as a 'fortuitous coincidence, since the omission of higher streamwise eigenfunctions is only possible in the presence of diffusion'. The 'coincidence' 
may be because in this limit the front is sharp relative to the perturbation lengthscale $m^{-1}$, and so can be approximated as the perfectly sharp interface assumed in [2]; similarly, the perturbations $\phi_{0}(\xi)$ and $\psi_{0}(\xi)$ correspond to infinitesimal advances or retardations of the front, which were the perturbations treated in the original analysis. We note, though, that the predictions of the current model deviate from those of Saffman \& Taylor [2] at higher order in $m$ as the dependence of $\mathrm{E}$ on $t$ becomes apparent.

\subsection{Properties of the eigenvalues $\sigma_{ \pm}$}

We are in general interested in the behaviour of the growth rates $\sigma_{ \pm}$in the regime $m \ll 1$. A first question which arises is whether complex values of $\sigma_{ \pm}$ are possible in this regime, giving an oscillatory instability (cf. [10], §5.3.2). The discriminant of $\sigma_{ \pm}$is given by

$$
\left(E_{11}-E_{22}\right)^{2}+4 E_{12} E_{21}=\frac{1}{4}\left(\beta_{C}+\beta_{T}\right)^{2} m^{2}+\mathcal{O}\left(m^{3}\right)
$$

so for sufficiently small $m$, the eigenvalues $\sigma_{ \pm}$are real and there is no reason to expect oscillatory instabilities.

To investigate the long-wave limit, it is instructive to expand $\sigma_{ \pm}$in powers of $m$. We find that

$$
\begin{aligned}
\sigma_{ \pm} & =\frac{\beta_{C}+\beta_{T}}{4} m \pm \frac{1}{4}\left[\left(\beta_{C}+\beta_{T}\right)^{2}\right]^{1 / 2} m+\mathcal{O}\left(m^{2}\right) \\
& \sim \frac{\beta_{C}+\beta_{T}}{4}\left(1 \pm \operatorname{sgn}\left(\beta_{C}+\beta_{T}\right)\right) m .
\end{aligned}
$$

This means that, if the total mobility contrast $\beta_{\text {tot }} \equiv \beta_{C}+\beta_{T}>0$, we have $\sigma_{+} \sim \frac{1}{2} \beta_{\text {tot }} m$ and $\sigma_{-}=\mathcal{O}\left(m^{2}\right)$, whereas if $\beta_{\text {tot }}<0$, we have $\sigma_{+}=\mathcal{O}\left(m^{2}\right)$ and $\sigma_{-} \sim \frac{1}{2} \beta_{\text {tot }} m$.

In the regime $0<\beta_{\text {tot }} \ll 1$, then, the front is unstable in the long-wave limit regardless of how the total mobility contrast $\beta_{\text {tot }}$ is made up. In the case $\beta_{\text {tot }}<0$, we need to consider terms of order $m^{2}$. Carrying out the expansion, we obtain, for $\beta_{\text {tot }}<0$,

$$
\sigma_{+}=\frac{-1}{\beta_{T}+\beta_{C}}\left[\beta_{T}+L e \beta_{C}+\beta_{T} \beta_{C} \sqrt{\frac{2 t}{\pi}}(1+\sqrt{L e}-\sqrt{2(1+L e)})\right] m^{2}+\mathcal{O}\left(m^{3}\right) .
$$

A long-wave instability will therefore occur if the quantity in square brackets is positive, i.e.

$$
\beta_{T}+L e \beta_{C}+\beta_{T} \beta_{C} \sqrt{\frac{2 t}{\pi}}(1+\sqrt{L e}-\sqrt{2(1+L e)})>0 .
$$


The quantity in round brackets is negative for all $L e>1$. We rewrite the instability condition as

$$
\beta_{T}+L e \beta_{C}>\beta_{T} \beta_{C} \sqrt{\frac{2 t}{\pi}}(\sqrt{2(1+L e)}-1-\sqrt{L e})
$$

If $\beta_{C}<0$ and $\beta_{T}<0$, the condition is trivially not satisfied since the LHS $<0$ while the RHS $>0$. If $\beta_{T} \beta_{C}<0$ (i.e. exactly one component is destabilising), the instability condition becomes

$$
\frac{1}{\beta_{C}}+\frac{L e}{\beta_{T}}<\sqrt{\frac{2 t}{\pi}}(\sqrt{2(1+L e)}-1-\sqrt{L e})
$$

This implies that if $1 / \beta_{C}+L e / \beta_{T} \leq 0$, the front becomes unstable immediately; while if $1 / \beta_{C}+L e / \beta_{T}>0$, the front becomes unstable only after a time $t_{\text {crit }}$ which is given by

$$
t_{\mathrm{crit}}=\frac{\pi\left(\beta_{T}+L e \beta_{C}\right)^{2}}{2 \beta_{T}^{2} \beta_{C}^{2}(\sqrt{2(1+L e)}-1-\sqrt{L e})^{2}} .
$$

This is reminiscent of the critical time required for a diffusively spreading thermal front in a porous medium to become gravitationally unstable: compare, for example, [20] or [21]. It is also reminiscent of the destabilisation of initially stable displacement processes with non-monotonic viscosity profiles predicted by [22] and by [18].

It is important to note that although the analysis we have presented shows that the front cannot become unstable before some critical time, we have not formally shown that such a critical time exists. This is because the critical time predicted by (47) is of order $\left|\beta_{T} \beta_{C}\right|^{-1} \gg 1$, and we have previously assumed that $t=\mathcal{O}(1)$. However, the result is at least suggestive. (Note that we have retained terms of order $\left|\beta_{T} \beta_{C}\right|$ in the expansion: as long as $\left|\beta_{C}\right|$ and $\left|\beta_{T}\right|$ are of comparable magnitude the retention of these terms is legitimate since the small-viscosity contrast approximation is valid to second order in $\left|\beta_{C}\right|+\left|\beta_{T}\right|$ : see appendix A for details.)

Figure 1 summarises the stability behaviour of the front as predicted by our analysis. It is instructive to compare the criteria obtained above with one of our heuristic arguments for the stability criterion, which is that instability should occur when the viscosity profile has an increasing region somewhere [18]. We recall that this occurs if $1<-\beta_{T} / \beta_{C}<\sqrt{L e}$ : this boundary is plotted as the dotted line in figure 1 , and we note that it does not provide a good guide to the stability of the front. 


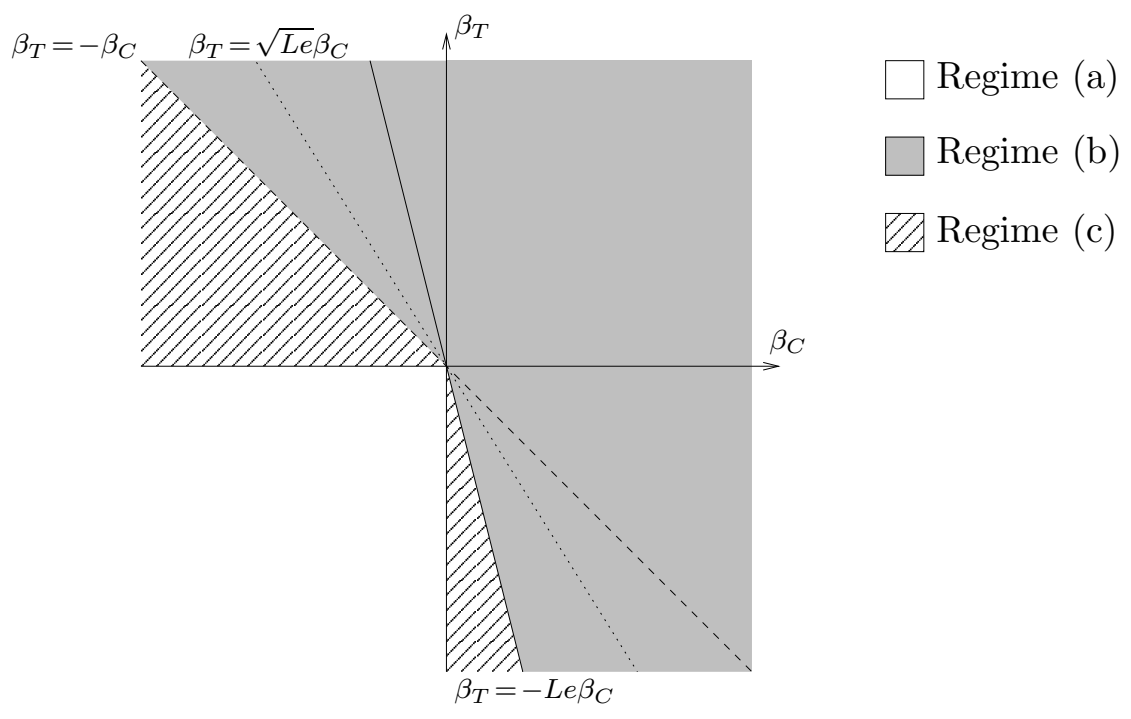

Fig. 1. Schematic showing the predicted stable and unstable regions of the $\left(\beta_{C}, \beta_{T}\right)$ plane for $L e>1, m \sqrt{t} \ll 1$ and $\left|\beta_{C}\right|,\left|\beta_{T}\right| \ll 1$. The dashed line represents $\beta_{T}+\beta_{C}=0$; the solid line represents $\beta_{T}+L e \beta_{C}=0$. The dotted line represents $\beta_{T}+\sqrt{L} e \beta_{C}=0$, i.e. the cases where an inflection point occurs in the viscosity profile. In regime (a) (white), the front remains stable for all time. In regime (b) (gray), the front becomes unstable immediately. In regime (c) (hatched), the front is stable at $t=0$ but may become unstable as it spreads.

\subsection{Discussion and interpretation}

In the limit $m \rightarrow 0$, the eigenvector corresponding to $\sigma_{+}$is given by

$$
\left(\frac{B}{A}\right)_{+}=\frac{\beta_{T}-\beta_{C}+\sqrt{\left(\beta_{C}+\beta_{T}\right)^{2}}}{2 \beta_{T}}+\mathcal{O}(m) .
$$

If $\beta_{C}+\beta_{T}>0$, then this simplifies to

$$
\left(\frac{B}{A}\right)_{+} \sim 1
$$

so the fastest-growing mode is a 'sinuous' perturbation (cf. [23]; [10], §3), with the temperature and concentration fields perturbed in the same sense. Note that in this regime, we can see from (32) that the velocity perturbation $U_{1}$ is of order $m$ in amplitude.

If $\beta_{C}+\beta_{T}<0$, the eigenvector simplifies to

$$
\left(\frac{B}{A}\right)_{+} \sim-\frac{\beta_{C}}{\beta_{T}}
$$

When $\beta_{C} / \beta_{T}<0$, so one component is destabilising, the dominant mode is again sinuous, although it is now 'lopsided', with one field perturbed more 
strongly than the other. From (32), we see that in this regime the velocity perturbation $U_{1}$ is of order $m^{2}$ in amplitude, significantly weaker than in the regime $\beta_{C}+\beta_{T}>0$. (In the double-stabilised regime $\beta_{C}<0$ and $\beta_{T}<0$, the most slowly-decaying mode is varicose, with the perturbations to temperature and concentration opposing each other and thus reducing the dynamic stabilisation of the perturbation through the Saffman-Taylor mechanism.)

We can now relate the findings of our analysis to the heuristic arguments proposed in $\S 1.1$. The argument (ii), that in the long-wave limit the growth rates are the same as for the immiscible case, applies to the $\mathcal{O}(m)$ term in the growth rate, which is unaffected by transverse diffusion: this is sufficient to provide an immediately unstable mode wherever $\beta_{T}+\beta_{C}>0$. There is also a second mode of perturbation, however, in addition to the single mode in the single-species case: in the regime $\beta_{C}>0,-L e \beta_{C}<\beta_{T}<-\beta_{C}$, this 'lopsided' mode is able to grow, although the velocity perturbation is weakened and the growth inhibited by the unfavourable thermal viscosity contrast. The growth of this secondary mode was not predicted by any of our heuristic arguments. The argument (iii), concerning the non-monotonicity of the viscosity profile, is seen not to be relevant in this regime, while the results for critical times suggest that argument (i) holds at large times (but we must confirm this by a separate analysis).

\section{Long-time asymptotics}

The other natural asymptotic limit to investigate is that of long time, when the front has spread considerably and — again following [16] — we may expect perturbations to be strongly localised so that they are in effect embedded within the front. The natural expansion variable is again $m \sqrt{t}$, and we consider the regime $m \sqrt{t} \gg 1$, this time assuming that the parameters $L e,\left|\beta_{T}\right|$ and $\left|\beta_{C}\right|$ are all of order unity.

Ben, Demekhin \& Chang [16] develop an asymptotic representation of $U_{1}$ in this limit by assuming that, if the perturbation to velocity $U_{1}$ is strongly localised about $\xi=0$ so that we need only consider values of $|\xi| \ll m \sqrt{t}$, then the term $\mathrm{e}^{-\xi^{2}} \mathrm{~d} U_{1} / \mathrm{d} \xi$ in equation (24) may be approximated simply by $\mathrm{d} U_{1} / \mathrm{d} \xi$. It is hard to justify this assumption, since the solution which they obtain decays as $\mathrm{e}^{-\xi^{2}}$, in other words at the same rate as the function which they expand about $\xi=0$. (Their statement that $m \sqrt{t} \gg \xi$ is formally meaningless, since the boundary conditions on $U_{1}$ must be applied at $\xi= \pm \infty$.) However, by taking a slightly different approach we can develop solutions in the regime $m \sqrt{t} \gg 1$ directly. 
We define the expansion variable $M=m \sqrt{t}$ and write equation (24) as

$$
\frac{\mathrm{d}^{2} U_{1}}{\mathrm{~d} \xi^{2}}+f(\xi) \frac{\mathrm{d} U_{1}}{\mathrm{~d} \xi}-4 M^{2} U_{1}=M^{2} g(\xi)
$$

where

$$
f(\xi)=\beta_{T} \frac{\mathrm{e}^{-\xi^{2} / L e}}{\sqrt{\pi L e}}+\beta_{C} \frac{\mathrm{e}^{-\xi^{2}}}{\sqrt{\pi}} \quad \text { and } \quad g(\xi)=\frac{2 \beta_{C}}{\sqrt{\pi t}} A(t) \mathrm{e}^{-\xi^{2}}+\frac{2 \beta_{T}}{\sqrt{\pi L e t}} B(t) \mathrm{e}^{-\xi^{2} / L e} .
$$

The important point is that $f(\xi)$ and $g(\xi)$ are both even in $\xi$ and independent of $M$.

We seek solutions of the form

$$
U_{1}(\xi)=U_{10}(\xi)+\frac{1}{M^{2}} U_{11}(\xi)+\mathcal{O}\left(\frac{1}{M^{4}}\right),
$$

and substituting this into equation (51) we obtain at $\mathcal{O}\left(M^{2}\right)$ and $\mathcal{O}(1)$ the equations

$$
U_{10}(\xi)=-\frac{1}{4} g(\xi) \quad \text { and } \quad \frac{\mathrm{d}^{2} U_{10}}{\mathrm{~d} \xi^{2}}+f(\xi) \frac{\mathrm{d} U_{10}}{\mathrm{~d} \xi}-4 U_{11}=0,
$$

giving us immediately the asymptotic solution

$$
U_{1} \sim-\frac{1}{4} g(\xi)-\frac{1}{16 M^{2}}\left[\frac{\mathrm{d}^{2} g}{\mathrm{~d} \xi^{2}}+f(\xi) \frac{\mathrm{d} g}{\mathrm{~d} \xi}\right]+\mathcal{O}\left(\frac{1}{M^{4}}\right) .
$$

(Note that because of the exponential decay in $f(\xi)$ and $g(\xi)$ these solutions satisfy the boundary conditions as $\xi \rightarrow \pm \infty$ : this contrasts with the situation in the small- $M$ limit where the expansions were not uniform in $\xi$.) The first term proportional to $M^{-2}$ is even in $\xi$, while the second term is odd. This means that when either of the inner products $\left\langle\phi_{0} U_{1}\right\rangle_{C}$ or $\left\langle\psi_{0} U_{1}\right\rangle_{T}$ is taken, only the first of these terms will contribute to the integral. Consequently, to $\mathcal{O}\left(M^{-2}\right)$, the form of $f(\xi)$ is irrelevant so long as it is of order unity and even in $\xi$. This explains the success of Ben et al.'s formally invalid approximation of equation (24): had the asymptotic series been extended to $\mathcal{O}\left(M^{-4}\right)$, or had we required odd terms in $\xi$, then the approximation would have failed.

Substituting in for $f(\xi)$ and $g(\xi)$, we have

$$
\begin{aligned}
U_{1}(\xi) \sim & -\frac{\beta_{C}}{2 \sqrt{\pi t}} A(t) \mathrm{e}^{-\xi^{2}}-\frac{\beta_{T}}{2 \sqrt{\pi L e t}} B(t) \mathrm{e}^{-\xi^{2} / L e} \\
& -\frac{1}{4 m^{2} t}\left[\frac{\beta_{C} A(t)}{\sqrt{\pi t}} \mathrm{e}^{-\xi^{2}}\left(2 \xi^{2}-1\right)+\frac{\beta_{T} B(t)}{L e^{3 / 2} \sqrt{\pi t}} \mathrm{e}^{-\xi^{2} / L e}\left(2 \frac{\xi^{2}}{L e}-1\right)\right. \\
& \left.-\left(\beta_{T} \frac{\mathrm{e}^{-\xi^{2} / L e}}{\sqrt{\pi L e}}+\beta_{C} \frac{\mathrm{e}^{-\xi^{2}}}{\sqrt{\pi}}\right)\left(\frac{\beta_{C} A(t)}{\sqrt{\pi t}} \xi \mathrm{e}^{-\xi^{2}}+\frac{\beta_{T} B(t)}{L e^{3 / 2} \sqrt{\pi t}} \xi \mathrm{e}^{-\xi^{2} / L e}\right)\right] .
\end{aligned}
$$


We can now calculate asymptotic estimates for the inner products:

$$
\begin{gathered}
\left\langle\mathrm{e}^{-\xi^{2}} U_{1}\right\rangle_{C}=\frac{A(t) \beta_{C}}{2 \sqrt{2 \pi t}}\left[-1+\frac{1}{4 m^{2} t}\right]+\frac{B(t) \beta_{T}}{2 \sqrt{1+L e} \sqrt{\pi t}}\left[\frac{1}{2 m^{2} t(1+L e)}-1\right] \\
\left\langle\mathrm{e}^{-\xi^{2} / L e} U_{1}\right\rangle_{T}=\frac{A(t) \beta_{C}}{2 \sqrt{1+L e} \sqrt{\pi t}}\left[\frac{1}{2 m^{2} t(1+L e)}-1\right]+\frac{B(t) \beta_{T}}{2 \sqrt{2 \pi L e t}}\left[\frac{1}{4 m^{2} L e t}-1\right] .
\end{gathered}
$$

The evolution equation becomes

$$
\frac{\mathrm{d}}{\mathrm{d} t}\left(\begin{array}{l}
A \\
B
\end{array}\right)=\left(\begin{array}{cc}
E_{11} & E_{12} \\
E_{21} & E_{22}
\end{array}\right) \cdot\left(\begin{array}{l}
A \\
B
\end{array}\right)
$$

where the components of the matrix $\mathrm{E}$ are given by

$$
\begin{gathered}
E_{11}=-m^{2}-\frac{\beta_{C}}{2 \sqrt{2 \pi t}}\left[-1+\frac{1}{4 m^{2} t}\right], \\
E_{12}=-\frac{\beta_{T}}{2 \sqrt{1+L e} \sqrt{\pi t}}\left[\frac{1}{2 m^{2} t(1+L e)}-1\right], \\
E_{21}=-\frac{\beta_{C}}{2 \sqrt{1+L e} \sqrt{\pi t}}\left[\frac{1}{2 m^{2} t(1+L e)}-1\right], \\
E_{22}=-m^{2} L e-\frac{\beta_{T}}{2 \sqrt{2 \pi L e t}}\left[-1+\frac{1}{4 m^{2} L e t}\right] .
\end{gathered}
$$

As before, the eigenvalues of $\mathrm{E}$, and thus the growth rates of perturbations, are given by

$$
\sigma_{ \pm}=\frac{1}{2}\left(E_{11}+E_{22}\right) \pm \frac{1}{2}\left[\left(E_{11}-E_{22}\right)^{2}+4 E_{12} E_{21}\right]^{1 / 2}
$$

while the structure of the corresponding perturbations is described by the ratio of the solute to the thermal perturbation amplitude,

$$
\left(\frac{B}{A}\right)_{ \pm}=\frac{E_{22}-E_{11} \pm \sqrt{\left(E_{11}-E_{22}\right)^{2}+4 E_{12} E_{21}}}{2 E_{12}} .
$$

\subsection{Reduction to the single-species case}

It is useful to consider briefly the case $\beta_{T}=0$, so the growth rate is given simply by $\sigma=E_{11}$. Again, this has the same form as the growth rate derived by Ben, Demekhin \& Chang [16] (their equation 50), but the coefficients 
are slightly different, apparently because of an algebraic error in Ben et al.'s equation (49).

It is worth extracting some information from this case before proceeding. The cut-off condition for marginal stability (assuming that the principle of exchange of stabilities holds) is given by

$$
E_{11}=0 \quad \Longleftrightarrow \quad m^{4}-\frac{\beta_{C}}{2 \sqrt{2 \pi} t^{1 / 2}} m^{2}+\frac{\beta_{C}}{8 \sqrt{2 \pi} t^{3 / 2}}=0 .
$$

This has solutions

$$
m^{2}=\left(m_{ \pm}^{e x}\right)^{2}=\frac{1}{2}\left[\frac{\beta_{C}}{2 \sqrt{2 \pi} t^{1 / 2}} \pm\left(\left(\frac{\beta_{C}}{2 \sqrt{2 \pi} t^{1 / 2}}\right)^{2}-4 \frac{\beta_{C}}{8 \sqrt{2 \pi} t^{3 / 2}}\right)^{1 / 2}\right]
$$

Concerning ourselves for the moment only with the case $\beta_{C}>0$, we may write these as

$$
\left(m_{ \pm}^{e x}\right)^{2}=\frac{\beta_{C}}{4 \sqrt{2 \pi} t^{1 / 2}}\left[1 \pm\left(1-\frac{2 \sqrt{2 \pi}}{\beta_{C}} \frac{1}{t^{1 / 2}}\right)^{1 / 2}\right]
$$

We therefore have different scalings with $t$ for the upper and lower boundaries of the range of unstable wavenumbers:

$$
\left(m_{+}^{e x}\right)^{2} \sim \frac{\beta_{C}}{2 \sqrt{2 \pi}} t^{-1 / 2} \quad \text { and } \quad\left(m_{-}^{e x}\right)^{2} \sim \frac{1}{4} t^{-1} .
$$

We conclude that the maximum unstable wavenumber scales as $m_{+}^{e x} \sim t^{-1 / 4}$ while the minimum unstable wavenumber scales as $m_{-}^{e x} \sim t^{-1 / 2}$.

Meanwhile, the wavenumber at which the maximally unstable perturbation occurs must be a stationary point, satisfying

$$
\frac{\mathrm{d} E_{11}}{\mathrm{~d} m}=0 \Longleftrightarrow 2 m=\frac{\beta_{C}}{2 \sqrt{2 \pi t}} \frac{1}{2 m^{3} t} \Longleftrightarrow m=m^{s t}=\frac{\beta_{C}^{1 / 4}}{2^{7 / 8} \pi^{1 / 8}} t^{-3 / 8} .
$$

A further scaling with $t$ is therefore involved, because the balance which determines the maximum growth rate is different from those that determine the cut-off wavelengths.

Note that an asymptotic expansion for the single-species problem that only preserved the leading-order terms in $t$ at any stage would be liable to fail, for example by predicting maximum growth rates at $m=0$. In the more complicated double-diffusive problem, where we cannot readily solve explicitly for cut-off and maximally unstable wavenumbers, some care is therefore required.

One manifestation of this care concerns the selection of the long-wave (small$m$ ) cut-off wavenumber. Recall that $\sigma$ was derived in the limit $m \sqrt{t} \rightarrow \infty$. 
This means that the asymptotic estimate for $m_{+}^{e x}$ is valid at large times, since $m_{+}^{e x} \sqrt{t} \sim t^{1 / 4} \rightarrow \infty$ as $t \rightarrow \infty$; similarly the asymptotic estimate for $m^{s t}$ is valid at large times, since $m^{s t} \sqrt{t} \sim t^{1 / 8} \rightarrow \infty$ as $t \rightarrow \infty$. However, the asymptotic estimate for $m_{-}^{e x}$ is not formally valid, since $m_{-}^{e x} \sqrt{t}=\mathcal{O}(1)$ as $t \rightarrow \infty$. We will therefore consider only those quantities the wavenumbers of which scale with $t^{-1 / 4}$ or $t^{-3 / 8}$, and accept that our expression for $\sigma$ will not provide a valid prediction of the long-wave cut-off wavenumber $m_{-}^{e x}$.

Our strategy will be to start with the short-wave end of the spectrum and look at features which scale as $m \sim t^{-1 / 4}$ at large times. We will then consider scalings with $m \sim t^{-3 / 8}$. It is helpful at this stage to introduce some shorthand notation: we will consider regimes of behaviour in the $\left(\beta_{C}, \beta_{T}\right)$-plane, and refer to the quadrants by the corresponding compass points, so that for example the $\mathrm{SE}$ quadrant is the region $\beta_{C}>0, \beta_{T}<0$.

\subsection{Long-time asymptotics: expansion capturing $t^{1 / 4}$ scalings}

We will start by trying to develop a consistent expansion in which the interesting values of $m$ scale with $t^{-1 / 4}$. We will try to extract information about the cut-off wavenumber(s) and the fastest-growing wavenumber, noting that if we predict a short- or long-wave catastrophe of any kind, this suggests that we can only obtain the relevant information from a different distinguished limit.

Formally, we consider the limit in which $t \rightarrow \infty$ while the newly-defined quantity $\mu=m t^{1 / 4}$ remains constant (i.e. of order unity). Substituting this into (61) and expanding for large $t$, we find

$$
\begin{gathered}
\sigma_{+}=\frac{\sigma_{1}}{t^{1 / 2}}+\mathcal{O}\left(\frac{1}{t}\right), \quad \text { where } \quad \sigma_{1}=-a \mu^{2}+b+\sqrt{\left(c \mu^{2}-d\right)^{2}+e}, \quad \text { for } \\
a=\frac{(L e+1)}{2}, \quad b=\frac{\beta_{T}+\beta_{C} \sqrt{L e}}{4 \sqrt{2 \pi L e}}, \quad c=\frac{(L e-1)}{2} \\
d=\frac{\left(\beta_{T}-\beta_{C} \sqrt{L e}\right)}{4 \sqrt{2 \pi L e}}, \quad e=\frac{\beta_{T} \beta_{C}}{4 \pi(L e+1)}
\end{gathered}
$$

Note that $\sigma_{+} \sim-\mu^{2}$ as $\mu \rightarrow \infty$, so large-wavenumber perturbations are always decaying. Whether unstable perturbations exist then depends on whether $\Re\left(\sigma_{+}\right)>0$ for any finite $\mu$.

\subsubsection{Behaviour of $\sigma_{1}$ as $\mu \rightarrow 0$}

The first bit of information to extract is how $\sigma_{1}$ behaves as $\mu \rightarrow 0$. (This is formally invalid, but provides a useful tool to determine the behaviour of 
$\sigma_{1}(\mu)$ for finite $\mu$.) In this limit we have

$$
\sigma_{1}(0)=b+\sqrt{d^{2}+e} .
$$

We first confirm that $\sigma_{1}(0)$ is always real. We may write

$$
d^{2}+e=\frac{\beta_{T}^{2}}{32 \pi L e}+\frac{\left(\beta_{C} \sqrt{L e}\right)^{2}}{32 \pi L e}+\frac{\beta_{T} \beta_{C} \sqrt{L e}}{32 \pi L e} \frac{2(4 \sqrt{L e}-1-L e)}{(L e+1)} .
$$

By the triangle inequality we know that $\beta_{T}^{2}+\beta_{C}^{2} L e>2\left|\beta_{T} \beta_{C} \sqrt{L e}\right|$; a further application of the triangle inequality then shows that

$$
\left|\frac{2(4 \sqrt{L e}-1-L e)}{(L e+1)}\right|<2 \text { for } L e>1
$$

and so we conclude that $d^{2}+e>0$ and the predicted value of $\sigma_{1}$ as $\mu \rightarrow 0$ is always real.

We now look at the sign of $\sigma_{1}(0)$. Clearly in the NE quadrant, where $b>0$, we must have $\sigma_{1}(0)>0$. We now look for places where $\sigma_{1}(0)=0$. We have

$$
\begin{aligned}
b+\sqrt{d^{2}+e}=0 & \Longrightarrow b<0 \text { and } b^{2}-d^{2}=e \\
& \Longrightarrow b<0 \text { and } \frac{\beta_{T} \beta_{C}}{4 \pi \sqrt{L e}}\left(\frac{1}{2}-\frac{\sqrt{L e}}{L e+1}\right)=0
\end{aligned}
$$

so, recalling that $L e>1$ so that the contents of the round brackets are nonzero, we see that $\sigma_{1}(0)$ can change sign only where $\beta_{T}=0$ or $\beta_{C}=0$. It remains to be determined in which quadrants $\sigma_{1}(0) \gtrless 0$. In the SW quadrant, the condition

$$
\begin{aligned}
b+\sqrt{d^{2}+e}<0, \quad b<0 & \Longleftrightarrow \frac{\beta_{C} \beta_{T}}{8 \pi \sqrt{L e}}>\frac{\beta_{C} \beta_{T}}{4 \pi(L e+1)} \\
& \Longleftrightarrow \frac{\sqrt{L e}}{L e+1}<\frac{1}{2} .
\end{aligned}
$$

Since this final condition holds for all $L e>1$, we conclude that $\sigma_{1}(0)<0$ throughout the SW quadrant.

Finally, we note that since $b<0 \Longleftrightarrow \beta_{T}<-\sqrt{L e} \beta_{C}$, the condition $\sigma_{1}(0)=0$ can be met only on the negative parts of the $\beta_{C}$ and $\beta_{T}$ axes, not on the upper parts. We conclude that $\sigma_{1}(0)<0$ within the SW quadrant, that $\sigma_{1}(0)>0$ within the other three quadrants, and that $\sigma_{1}(0)=0$ on the axes $\beta_{C}=0$ and $\beta_{T}=0$. (This latter is unsurprising: if $\beta_{C}=0$ or $\beta_{T}=0$ then one component does not affect the flow, so we are free to perturb this component by an infinitesimal advance or retreat which will then not decay - the very basis on which we have focused our attention on the leading-order streamwise structure of the perturbation.) 
We will now examine the behaviour for $\mu>0$, proceeding quadrant-byquadrant.

\subsubsection{Behaviour of $\sigma_{1}$ in the $N E$ and $S W$ quadrants: $\beta_{C} \beta_{T}>0$}

In these quadrants the behaviour of $\sigma_{1}(\mu)$ is fairly easy to establish. Note that we have $e>0$, so $\sigma_{1} \in \mathbb{R} \forall \mu \geq 0$. We now look for possible local maxima over $\mu$. We have

$$
\frac{\mathrm{d} \sigma_{1}}{\mathrm{~d} \mu}=\frac{-2 \mu\left[a \sqrt{\left(c \mu^{2}-d\right)^{2}+e}-c\left(c \mu^{2}-d\right)\right]}{\sqrt{\left(c \mu^{2}-d\right)^{2}+e}} .
$$

One stationary point therefore occurs at $\mu=0$, and seeking others we have the condition

$$
a \sqrt{\left(c \mu^{2}-d\right)^{2}+e}=c\left(c \mu^{2}-d\right) .
$$

There are then no real solutions with $\mu^{2}<d / c$; if $\mu^{2}>d / c$ then this equation implies

$$
\left(c \mu^{2}-d\right)^{2}\left(a^{2}-c^{2}\right)=-e a^{2}<0 .
$$

Since $a>c>0$ for all $L e>1$, we conclude that in these quadrants the only stationary point of $\sigma_{1}(\mu)$ occurs at $\mu=0$. This must then be the global maximum for $\mu \geq 0$ because of the behaviour we have already established in the large- $\mu$ limit.

We conclude that in these quadrants a 'long-wave catastrophe' is predicted when this asymptotic limit is considered: in fact, we will have to pursue the $t^{-3 / 8}$ expansion to locate the maximum. Two questions remain: is the maximal growth rate positive or negative, and is there a well-defined range of growing wavenumbers?

We deal with the maximal growth rate first. In the NE quadrant, we have $b>0$ and so $\sigma_{1}(0)=b+\sqrt{d^{2}+e}>0$, providing a well-defined and positive maximum growth rate. In the SW quadrant, $b<0$, so equation (69) confirms that $\sigma_{1}(0)<0$ and thus that everywhere in the SW quadrant all wavenumbers of perturbations are decaying (as physical intuition would suggest).

Now we consider whether there is a well-defined range of growing wavenumbers in the NE quadrant. The condition for exchange of stabilities, $\sigma_{1}=0$, becomes

$$
\begin{aligned}
& a \mu^{2}-b=\sqrt{\left(c \mu^{2}-d\right)^{2}+e}, \\
& \text { and so }\left\{\begin{array}{lll}
\left(a \mu^{2}-b\right)^{2}=\left(c \mu^{2}-d\right)^{2}+e & \text { if } & a \mu^{2}-b>0, \\
\text { no solutions exist } & \text { if } & a \mu^{2}-b<0 .
\end{array}\right.
\end{aligned}
$$

The equation $\left(a \mu^{2}-b\right)^{2}=\left(c \mu^{2}-d\right)^{2}+e$ becomes a quadratic in the variable 
$\mu^{2}$

$$
\mu^{4}+\frac{\left(-\beta_{T}-L e^{3 / 2} \beta_{C}\right)}{2 \sqrt{2} \sqrt{\pi} L e^{3 / 2}} \mu^{2}+\frac{\beta_{C} \beta_{T}(\sqrt{L e}-1)^{2}}{8 \pi(L e+1) L e^{3 / 2}}=0
$$

which has solutions

$$
\mu^{2}=\left(\mu_{ \pm}^{e x}\right)^{2}=\frac{\left(\beta_{T}+L e^{3 / 2} \beta_{C}\right)}{4 \sqrt{2} \sqrt{\pi} L e^{3 / 2}} \pm \sqrt{\frac{\left(\beta_{T}+L e^{3 / 2} \beta_{C}\right)^{2}}{\left(4 \sqrt{2} \sqrt{\pi} L e^{3 / 2}\right)^{2}}-\frac{\beta_{C} \beta_{T}(\sqrt{L e}-1)^{2}}{8 \pi(L e+1) L e^{3 / 2}}}
$$

Either of $\mu_{ \pm}^{e x}$ will provide exactly one real positive root for $\mu$, if and only if $\left(\mu_{ \pm}^{e x}\right)^{2}>\max (0, b / a)$. Since $\sigma_{1}(\mu)$ is monotonically decreasing, is positive for $\mu=0$ and becomes negative for large $\mu$, there can only be exactly one root of the equation $\sigma_{1}(\mu)=0$, and this root must be $\mu=\mu_{+}^{e x}$, since if $\left(\mu_{-}^{e x}\right)^{2}>b / a$ then $\left(\mu_{+}^{e x}\right)^{2}>b / a$ and we would have two roots and a contradiction.

We conclude that in the NE quadrant there is a well-defined set of unstable wavenumbers satisfying $\mu<\mu_{+}^{e x}$; in other words, the shortest unstable wavenumber satisfies $m \sim \mu_{+}^{e x} t^{-1 / 4}$.

\subsubsection{Behaviour of $\sigma_{1}$ in the $N W$ and SE quadrants: $\beta_{C} \beta_{T}<0$}

In these quadrants $e<0$, so it is no longer immediately apparent that $\sigma_{1}(\mu) \in$ $\mathbb{R} \forall \mu>0$.

The condition $\sigma_{1} \notin \mathbb{R}$ corresponds to

$$
\left(c \mu^{2}-d\right)^{2}+e<0 \Longleftrightarrow \frac{d-\sqrt{-e}}{c}<\mu^{2}<\frac{d+\sqrt{-e}}{c},
$$

where we have used the fact that $e<0$ in these quadrants.

In the NW quadrant, where $\beta_{C}<0$ and $\beta_{T}>0, d>0$ and so the range of wavenumbers where $\sigma_{1} \notin \mathbb{R}$ includes at least some positive values of $\mu^{2}$. (Note that from above we know that $d^{2}+e>0$; when $d>0$ this means we can conclude that $d-\sqrt{-e}>0$, so the condition (82) must define a band of strictly positive values of $\mu^{2}$.)

In the SE quadrant, where $\beta_{C}>0$ and $\beta_{T}<0, d<0$. Again using the result that $d^{2}+e>0$ so $|d|>\sqrt{-e}$, we conclude that the condition (82) cannot be satisfied for any $\mu^{2}>0$; hence in this quadrant all values of $\sigma_{1}$ are real.

The SE quadrant: $\beta_{C}>0$ and $\beta_{T}<0$. We will look first at the SE quadrant. 
First we will look for possible local minima and maxima. As before, we have

$$
\frac{\mathrm{d} \sigma_{1}}{\mathrm{~d} \mu}=0 \quad \Longleftrightarrow \quad \mu=0 \quad \text { or } \quad a \sqrt{\left(c \mu^{2}-d\right)^{2}+e}=c\left(c \mu^{2}-d\right) .
$$

The latter equation has no solutions if $\mu^{2}<d / c$; however, since $d<0$ in this quadrant this criterion never comes into play, and the sole condition required for the validity of a root is that $\mu^{2}>0$. So we have

$$
a \sqrt{\left.\left(c \mu^{2}\right)-d\right)^{2}+e}=c\left(c \mu^{2}-d\right) \Longleftrightarrow \mu^{2}=\left(\mu_{ \pm}^{s t}\right)^{2}=\frac{d}{c} \pm \frac{1}{c} \sqrt{\frac{-e a^{2}}{a^{2}-c^{2}}}
$$

Since $d<0$, the root $\left(\mu_{-}^{s t}\right)^{2}<0$ and this is never a valid solution. So we have one of two situations: either $\left(\mu_{+}^{s t}\right)^{2}<0$, and the only stationary point of $\sigma_{1}$ is at $\mu=0$, or $\left(\mu_{+}^{s t}\right)^{2}>0$ and there is a second stationary point at $\mu=\mu_{+}^{s t}$ (which must be a local maximum because we know that $\sigma_{1}$ decays with $\mu$ for large $\mu$ ). The corresponding maximal growth rate is then given by

$$
\sigma_{1}\left(\mu_{+}^{s t}\right)=\frac{L e^{3 / 2} \beta_{C}-\beta_{T}}{2 \sqrt{2 \pi} \sqrt{L e}(L e-1)}-\frac{1}{\sqrt{\pi}(L e-1)} \sqrt{-\frac{\beta_{C} \beta_{T} L e}{L e+1}} .
$$

To determine where this applies, we look for the limits of the region where $\left(\mu_{+}^{s t}\right)^{2}>0$. The condition $\mu_{+}^{s t}=0$ becomes

$$
d+\sqrt{\frac{-e a^{2}}{a^{2}-c^{2}}}=0 \quad \Longleftrightarrow \quad \beta_{T}^{2}+(2 L e-2 \sqrt{L e}+2) \beta_{C} \beta_{T}+\beta_{C}^{2} L e=0 .
$$

This in turn has the solutions

$$
\beta_{T}=\beta_{T \pm}=\left[-1+\sqrt{L e}-L e \pm \sqrt{(-1+\sqrt{L e}-L e)^{2}-L e}\right] \beta_{C} .
$$

The contents of the square root sign are positive, and the contents of the square brackets are negative, for all $L e>1$; consequently both values of $\beta_{T}$ predicted for a given $L e>1$ and $\beta_{C}>0$ lie in the SE quadrant.

The behaviour of $\sigma_{1}$ in the SE quadrant is therefore as follows.

- If $\beta_{T}<\beta_{T-}\left(L e, \beta_{C}\right)$ or $\beta_{T}>\beta_{T+}\left(L e, \beta_{C}\right)$, then the global maximum occurs at $\mu=0$ and there are no local maxima; noting that $\sigma_{1}(0)>0$, the picture here is then the same as in the NE quadrant, with a long-wave 'catastrophe' and a cut-off wavenumber $\mu=\mu_{+}^{e x}$ for unstable perturbations.

- If $\beta_{T-}\left(L e, \beta_{C}\right)<\beta_{T}<\beta_{T+}\left(L e, \beta_{C}\right)$, then the stationary point at $\mu=$ 0 is a local minimum, the global maximum occurs at $\mu=\mu_{+}^{s t}>0$, and perturbations with $\mu>\mu_{+}^{e x}$ are stabilised. 
The NW quadrant: $\beta_{C}<0$ and $\beta_{T}>0$. In this quadrant, $a>c>1$ as before, $d>0, e<0$, and $b$ can be positive or negative. Recall that the key feature of this quadrant is that there is a range of wavenumbers, given by (82), in which $\sigma_{1} \notin \mathbb{R}$. Perturbations within this range are overdamped and may oscillate in sign while either growing or decaying (cf. [10], §5.3.2).

We will first seek stationary points of $\Re\left(\sigma_{1}(\mu)\right)$. If $\mu$ is within the range where $\sigma_{1} \notin \mathbb{R}$, then $\Re\left(\sigma_{1}\right)=-a \mu^{2}+b$, so $\mathrm{d} \Re\left(\sigma_{1}\right) / \mathrm{d} \mu=-2 a \mu<0$. Consequently we can never find a maximally growing perturbation with some oscillatory behaviour (unless the choice of values of $\mu$ is somehow restricted, for example by lateral boundaries to the flow).

If $\mu$ is within the range where $\sigma_{1} \in \mathbb{R}$, then we may proceed as before. There are stationary points at $\mu=0$ and where $a \sqrt{\left(c \mu^{2}-d\right)^{2}+e}=c\left(c \mu^{2}-d\right)$, and the condition for a solution to be valid is now that $\mu^{2}>d / c>0$. Of the two solutions to this equation, only one satisfies this condition, and it is given by

$$
\mu^{2}=\left(\mu_{+}^{s t}\right)^{2}=\frac{d}{c}+\frac{1}{c} \sqrt{\frac{-e a^{2}}{a^{2}-c^{2}}} .
$$

We must therefore consider whether this local maximum, which occurs for higher values of $\mu$ than the range defined by (82), can ever dominate the maximum at $\mu=0$.

Substituting $\mu=\mu_{+}^{s t}$ into $\sigma_{1}$, we find

$$
\sigma_{1}\left(\mu_{+}^{s t}\right)=\frac{b c-a d}{c}-\frac{\sqrt{-e\left(a^{2}-c^{2}\right)}}{c} .
$$

Now

$$
b c-a d=\frac{L e^{3 / 2} \beta_{C}-\beta_{T}}{4 \sqrt{2 \pi L e}}<0 \quad \text { in this quadrant. }
$$

Meanwhile, we recall that $\sigma_{1}(0)>0$ in this quadrant, so $\mu=0$ remains the global maximum predicted by this asymptotic expansion. (In reality, as usual, this means we must refer to a different asymptotic expansion to locate the minimum.)

We now look for the cut-off wavenumber $\mu=\mu_{\text {crit }}$ at which $\Re\left(\sigma_{1}\right)=0$. The expression which determines this will depend on whether $\sigma_{1}\left(\mu_{\text {crit }}\right)$ is real or not: if $\sigma_{1}\left(\mu_{\text {crit }}\right) \in \mathbb{R}$ then $\mu_{\text {crit }}=\mu_{+}^{e x}$ defined by (81) (which is valid as long as $\left.\left(\mu_{+}^{e x}\right)^{2}>b / a\right)$; while if $\sigma_{1}\left(\mu_{c r i t}\right) \notin \mathbb{R}$ then $\Re\left(\sigma_{1}\right)=-a \mu^{2}+b$ and so $\mu_{c r i t}^{2}=b / a$. Since $\Re\left(\sigma_{1}\right)$ is a continuous function and we have already shown that there is only one root, $\mu_{\text {crit }}$ must vary continuously with the parameters of the problem. This means that the boundaries of the region where the exchange of 
stabilities holds are the same as the boundaries of the region where $\mu=\mu_{+}^{e x}$ is a valid solution of $\sigma_{1}=0$. These boundaries are given by

$$
\beta_{T}=\beta_{T \pm}^{e x}=L e\left[-(L e+1-\sqrt{L e}) \pm \sqrt{(L e+1-\sqrt{L e})^{2}-L e}\right] \beta_{C}=L e \beta_{T \pm}
$$

where the last stage can be checked by referring to (87).

The behaviour of $\sigma_{1}$ in the NW quadrant is therefore as follows.

- Everywhere in this sector the highest growth rate occurs for $\mu \rightarrow 0$, i.e. we need to consider different asymptotics to pick out the fastest-growing wavenumber.

- If $L e \beta_{T+}<\beta_{T}<L e \beta_{T-}$ then the principle of exchange of stabilities does not hold: here the marginally unstable perturbations become unstable through an overdamped instability, and the cut-off wavenumber is $\mu=\sqrt{b / a}$. (Note that the line $b=0$, i.e. $\beta_{T}=-\sqrt{L e} \beta_{C}$, lies below the boundaries of this sector.)

- If $\beta_{T}<L e \beta_{T+}$ or $\beta_{T}>L e \beta_{T-}$ then the principle of exchange of stabilities does hold, and the cut-off wavenumber is $\mu=\mu_{+}^{e x}$ as before.

\subsection{Long-time asymptotics: expansion capturing $t^{3 / 8}$ scalings}

The analysis in the previous section suggests that, except in a particular sector within the SE quadrant, the scaling $m \sim t^{-1 / 4}$ fails to capture the maximally unstable wavenumber. Motivated by this and by the scaling of the maximum in the single-species case, we now consider features of $\sigma_{+}$whose location scales as $m \sim t^{-3 / 8}$.

We substitute into $\sigma_{+}$the ansatz $m=\nu t^{-3 / 8}$ and expand for large $t$, assuming $\nu=\mathcal{O}(1)$. This gives

$$
\sigma \sim \frac{\sigma_{1 \nu}}{t^{1 / 2}}+\frac{\sigma_{2 \nu}}{t^{3 / 4}}+\mathcal{O}\left(t^{-1}\right)
$$

where

$$
\sigma_{1 \nu}=\frac{\beta_{T}+\sqrt{L e} \beta_{C}}{4 \sqrt{2 \pi} \sqrt{L e}}+\sqrt{\left(\frac{\beta_{T}-\sqrt{L e} \beta_{C}}{4 \sqrt{2 \pi} \sqrt{L e}}\right)^{2}+\frac{\beta_{C} \beta_{T}}{4 \pi(L e+1)}}
$$

and $\sigma_{2 \nu}=p \nu^{2}+q / \nu^{2}$, where

$$
p=-\frac{(L e+1)}{2}+\left[\left(\beta_{T}-\sqrt{L e} \beta_{C}\right)^{2}+\frac{8 L e \beta_{T} \beta_{C}}{L e+1}\right]^{-1 / 2} \frac{(L e-1)}{2}\left(-\beta_{T}+\sqrt{L e} \beta_{C}\right)
$$


and

$$
\begin{aligned}
& q=-\left[\frac{\beta_{T}+L e^{3 / 2} \beta_{C}}{16 \sqrt{2 \pi} L e^{3 / 2}}\right]+\left[\left(\beta_{T}-\sqrt{L e} \beta_{C}\right)^{2}+\frac{8 L e \beta_{T} \beta_{C}}{L e+1}\right]^{-1 / 2} \times \\
& {\left[-\frac{\left(\beta_{T}+L e \beta_{C}\right)^{2}}{16 \sqrt{2 \pi} L e^{3 / 2}}+\frac{\beta_{T} \beta_{C}}{16 \sqrt{2 \pi}}\left(\frac{L e+1}{L e}+2 \frac{\left(1+L e^{2}-6 L e\right)}{\sqrt{L e}(L e+1)^{2}}\right)\right] . }
\end{aligned}
$$

Note that $\sigma_{1 \nu}$ is independent of the wavenumber, so we immediately obtain a leading-order estimate of the maximum growth rate, and this estimate is identical to the estimate of $\sigma(0)$ obtained using the $m=\mu t^{-1 / 4}$ scaling, equation (69). Crucially, we also inherit the result that this growth rate is real, $\sigma_{1 \nu} \in \mathbb{R}$. The same result shows that the contents of the square root sign in $\sigma_{2 \nu}$ are always non-negative, so $\sigma_{2 \nu}$ is also real.

To locate the maximum, we need to consider the next-order term, $\sigma_{2 \nu}$. There will be a global maximum corresponding to $\mathrm{d} \sigma_{2 \nu} / \mathrm{d} \nu=0$ if and only if $p<$ 0 and $q<0$. Some intricate but straightforward algebra (omitted here for clarity) indicates that $q=0$ only if $\beta_{C}=0$ or $\beta_{T}=0$, and sampling points in the four quadrants then confirms that $q>0$ in the SW quadrant $\left(\beta_{C}<0\right.$, $\left.\beta_{T}<0\right)$ and $q<0$ everywhere else. Since we expect growth rates to be negative in the SW quadrant in any case we will not worry further about the behaviour here. Similarly, solving $p=0$ and sampling shows that $p<0$ everywhere except in the sector of the SE quadrant bounded by the values $\beta=\beta_{T \pm}\left(\beta_{C}\right)$ defined in (87). We conclude that in the three quadrants where growth is possible, we can locate a well-defined maximum growth rate at a wavenumber which scales either with $t^{-3 / 8}$ (outwith the 'anomalous' sector) or with $t^{-1 / 4}$ (within the 'anomalous' sector).

Outwith the SW quadrant and the 'anomalous' sector, then, the maximum growth rate is therefore predicted to occur at

$$
\nu=\nu^{s t} \sim\left(\frac{q}{p}\right)^{1 / 4}, \quad \text { where } \quad \sigma=\sigma_{\max } \sim \frac{\sigma_{1 \nu}}{t^{1 / 2}}+\frac{2(p q)^{1 / 2}}{t^{3 / 4}} .
$$

\subsection{Summary of long-term asymptotic behaviour}

Figure 2 summarises the findings of the asymptotic analysis presented in the previous sections. For convenience we have also marked on the regime boundaries from figure 1: their position relative to the other boundaries is valid for all $L e>1$, though it should be remembered that they are strictly valid only for $\left|\beta_{C, T}\right| \ll 1$.

The $\left(\beta_{C}, \beta_{T}\right)$ parameter space divides into seven sectors, with five distinct regimes of long-time behaviour available. 


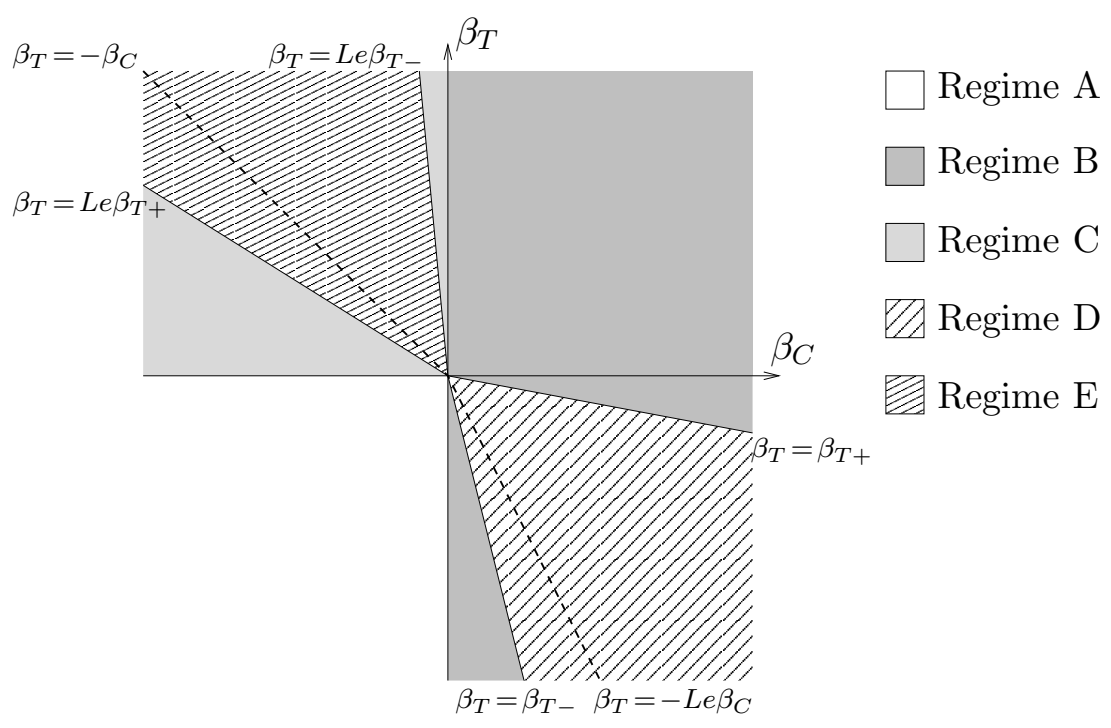

Fig. 2. Schematic of the stability regions in the $\left(\beta_{C}, \beta_{T}\right)$-plane, as predicted by the analyses using $m=\mu t^{-1 / 4}$ and $m=\nu t^{-3 / 8}$. The dashed lines represent the regime boundaries in the small- $m$, intermediate-time regime $(\S 4)$. See text for discussion of regimes.

Regime A. In this regime, the growth rates are always real and negative: the analysis with $m=\mu t^{-1 / 4}$ gives $\sigma_{1}<0 \forall \mu$, while the analysis with $m=\nu t^{-3 / 8}$ breaks down. Because the viscosity contributions from both components are negative, in this regime more viscous fluid is displacing less viscous fluid and there is no fingering instability.

Regime B. In this regime, the growth rates are always real. There is a cut-off wavenumber $m \sim \mu_{+}^{e x} t^{-1 / 4}$ such that all perturbations with higher wavenumbers than this are stable. The maximum growth rate is positive, and occurs at $m=\nu^{s t} t^{-3 / 8}$. Although there may be a small- $m$ stability cut-off, the asymptotic analysis presented here is not able to capture it.

Regime C. In this regime, it is possible for the growth rate to be complex for some wavenumbers, but this does not affect either the location of the cutoff wavenumber or the wavenumber corresponding to maximum growth rate; these are the same as in regime B. All perturbations with $m \gtrsim \mu_{+}^{e x} t^{-1 / 4}$ decay, but the decay may be oscillatory in nature. Although there may be a small- $m$ stability cut-off, the asymptotic analysis presented here is not able to capture it.

Regime D. In this regime, the growth rate is always real and there is a cut-off wavenumber $m \sim \mu_{+}^{e x} t^{-1 / 4}$ such that all perturbations with higher wavenumbers than this are stable. The maximum growth rate is positive, and occurs at $m \sim \mu^{s t} t^{-1 / 4}$. Although there may be a small- $m$ stability cut-off, the asymptotic analysis presented here is not able to capture it. 
Regime E. In this regime, there is a cut-off wavenumber $m \sim(b / a)^{1 / 2} t^{-1 / 4}$ such that all perturbations with higher wavenumbers than this are stable; at and around this wavenumber overdamped (oscillatory) perturbations occur. The fastest-growing perturbations have $m=\nu^{s t} t^{-3 / 8}$, and their growth rate is real so they do not exhibit overdamped behaviour. Although there may be a small- $m$ stability cut-off, the asymptotic analysis presented here is not able to capture it.

\subsection{Discussion and interpretation}

\subsubsection{Structure of dominant perturbations}

Substituting $m=\nu t^{-3 / 8}$ into the expression for $(B / A)_{+}$and expanding for large $t$, we obtain

$$
\left(\frac{B}{A}\right)_{+}=\frac{\sqrt{L e+1}}{2 \sqrt{2 L e} \beta_{T}}\left[\beta_{T}-\sqrt{L e} \beta_{C}+\sqrt{\left(\beta_{T}-\sqrt{L e} \beta_{C}\right)^{2}+\frac{8 L e \beta_{T} \beta_{C}}{\sqrt{L e+1}}}\right]+\mathcal{O}\left(\frac{1}{t^{1 / 4}}\right) .
$$

We find that $(B / A)_{+}>0$ in all except the SW quadrant, so wherever growing dominant modes with this scaling are possible they are sinuous perturbations.

Substituting $m=\mu_{+}^{s t} t^{-1 / 4}$ into the expression for $(B / A)_{+}$and expanding for large $t$, we obtain

$$
\left(\frac{B}{A}\right)_{+}=\sqrt{-\frac{\beta_{C}}{L e \beta_{T}}}+\mathcal{O}\left(\frac{1}{t^{1 / 2}}\right)
$$

where we have used the fact that this expression is relevant only in the SE quadrant where $\beta_{C}>0$ and $\beta_{T}<0$. We conclude that the preferred mode of instability here is again a 'lopsided' sinuous perturbation, so the dominant perturbations everywhere in parameter space are sinuous in this asymptotic limit. These results agree with those of Pritchard [10], who found that in the radial case sinuous perturbations were preferred even when the viscosity contributions of the two components were opposed.

\subsubsection{Physical interpretation of growth rates}

In dimensional form each entry in the evolution matrix $E$ has the dimension of a growth rate (i.e. inverse time). We can write these dimensional quantities 
as follows:

$$
\begin{aligned}
& \hat{E}_{11}=-\frac{\hat{\kappa}_{C}}{\hat{\lambda}^{2}}+\frac{1}{2 \sqrt{\pi}} \frac{\beta_{C} \hat{u}_{0}}{\left(2 \hat{\kappa}_{C} \hat{t}\right)^{1 / 2}}-\frac{1}{4 \sqrt{\pi}} \frac{\beta_{C} \hat{u}_{0} \hat{\lambda}^{2}}{\left(2 \hat{\kappa}_{C} \hat{t}\right)^{3 / 2}}, \\
& \hat{E}_{12}=\frac{1}{2 \sqrt{\pi}} \frac{\beta_{T} \hat{u}_{0}}{\left(\left(\hat{\kappa}_{C}+\hat{\kappa}_{T}\right) \hat{t}\right)^{1 / 2}}-\frac{1}{4 \sqrt{\pi}} \frac{\beta_{T} \hat{u}_{0} \hat{\lambda}^{2}}{\left(\left(\hat{\kappa}_{C}+\hat{\kappa}_{T}\right) \hat{t}\right)^{3 / 2}}, \\
& \hat{E}_{21}=\frac{1}{2 \sqrt{\pi}} \frac{\beta_{C} \hat{u}_{0}}{\left(\left(\hat{\kappa}_{C}+\hat{\kappa}_{T}\right) \hat{t}\right)^{1 / 2}}-\frac{1}{4 \sqrt{\pi}} \frac{\beta_{C} \hat{u}_{0} \hat{\lambda}^{2}}{\left(\left(\hat{\kappa}_{C}+\hat{\kappa}_{T}\right) \hat{t}\right)^{3 / 2}}, \\
& \hat{E}_{22}=-\frac{\hat{\kappa}_{T}}{\hat{\lambda}^{2}}+\frac{1}{2 \sqrt{\pi}} \frac{\beta_{T} \hat{u}_{0}}{\left(2 \hat{\kappa}_{T} \hat{t}\right)^{1 / 2}}-\frac{1}{4 \sqrt{\pi}} \frac{\beta_{T} \hat{u}_{0} \hat{\lambda}^{2}}{\left(2 \hat{\kappa}_{T} \hat{t}\right)^{3 / 2}} .
\end{aligned}
$$

Here $\hat{\lambda}=1 / \hat{m}$ is the wavelength of the perturbation.

To interpret these terms, we note the following points. First, since isotropic diffusion tends to favour structures with comparable lengthscales in both directions, the streamwise and transverse extents of an incipient finger (a localised perturbation to the variables) are both of order $\hat{\lambda}$. Second, in this regime the condition $m \sqrt{t} \gg 1$ means that the perturbation is embedded within the 'front' region over which the viscosity changes. This means that instead of being driven by the total viscosity contrasts $\left(\hat{\mu}_{1}-\hat{\mu}_{0}\right) / \hat{\mu}_{0} \sim \beta_{C, T}$ associated with each component, the perturbation is exposed to a fractional contrast $\Delta \mu_{C, T} \sim \beta_{C, T} \hat{\lambda} /\left(\hat{\kappa}_{C, T} \hat{t}\right)^{1 / 2}$. Third, the classic scaling for the growth rate associated with the destabilising Saffman-Taylor mechanism is $\hat{\sigma} \sim \Delta \mu \hat{u}_{0} / \hat{\lambda}$ (cf. equation (39)).

Three distinct scalings are present in the terms in equations (99): each may be interpreted as the contribution of a different physical effect, and we will consider each in turn. (It is interesting to compare the results of the heuristic analysis by Loggia et al. [22], which also found that the effects of different mechanisms combined additively in the long-wave limit: note, however, that Loggia et al.'s results contained only the first and second of the three types of term present in our analysis.)

The first terms in both $E_{11}$ and $E_{22}$ are straightforward to interpret: they represent the stabilising influences of solutal and thermal diffusion respectively. We will label terms of this type

$$
\hat{\sigma}_{\text {diff }} \propto \frac{\hat{\kappa}}{\hat{\lambda}^{2}}
$$

where $\hat{\kappa}$ may represent $\hat{\kappa}_{C}, \hat{\kappa}_{T}$ or some suitable combination of these, depending on context.

The second terms in $E_{11}$ and $E_{22}$ and the first terms in $E_{12}$ and $E_{21}$ all scale 


$$
\hat{\sigma}_{\mathrm{ST}} \propto \frac{\beta \hat{u}_{0}}{(\hat{\kappa} \hat{t})^{1 / 2}} \sim \frac{\Delta \mu \hat{u}_{0}}{\hat{\lambda}} .
$$

As the notation suggests, these represent the destabilising Saffman-Taylor mechanism, adjusted to allow for the fact that the perturbations are 'embedded' within the diffusing front.

The final terms in each of (99) have the form

$$
\hat{\sigma}_{\text {tran }} \propto \frac{\beta \hat{u}_{0} \hat{\lambda}^{2}}{(\hat{\kappa} \hat{t})^{3 / 2}}=\frac{\Delta \mu \hat{\lambda}}{(\hat{\kappa} \hat{t})^{1 / 2}} \times \frac{\hat{u}_{0} \hat{\lambda}}{(\hat{\kappa} \hat{t})^{1 / 2}} \times \frac{1}{\hat{\lambda}}
$$

The first factor represents the scaled mobility gradient in the transverse direction (which is related to that in the streamwise direction by a factor corresponding to the aspect ratio $\hat{\lambda} / \sqrt{\hat{\kappa} \hat{t}}$; the second factor is the transverse velocity obtained by continuity, $\partial \hat{u} / \partial \hat{x}=\partial \hat{v} / \partial \hat{y}$ and hence $\hat{v} \sim \hat{\lambda} \hat{u}_{0} / \sqrt{\hat{\kappa} \hat{t}}$; and the third factor is the perimeter of the finger (through which flux occurs) divided by the area into which this flux occurs. These terms, then, represent the transverse flow into a finger which is required because of the variation of streamwise velocity along that finger: this brings in less mobile fluid from the sides and tends to dilute and stabilise the perturbation.

Equipped with these scalings, we can demonstrate how the characteristic scales for the cut-off and favoured wavenumbers arise. A balance between $\hat{\sigma}_{\text {ST }}$ and $\hat{\sigma}_{\text {diff gives }}$

$$
\frac{\beta \hat{u}_{0}}{(\hat{\kappa} \hat{t})^{1 / 2}} \sim \frac{\hat{\kappa}}{\hat{\lambda}^{2}} \quad \text { and thus } \quad \hat{\lambda}^{2} \sim \frac{\hat{\kappa}^{3 / 8} \hat{t}^{1 / 4}}{\beta^{1 / 2} \hat{u}_{0}^{1 / 2}}
$$

it is this balance that controls the scaling of the cut-off wavenumber for short waves. A balance between $\hat{\sigma}_{\mathrm{ST}}$ and $\hat{\sigma}_{\text {tran }}$ gives

$$
\frac{\beta \hat{u}_{0}}{(\hat{\kappa} \hat{t})^{1 / 2}} \sim \frac{\beta \hat{u}_{0} \hat{\lambda}^{2}}{(\hat{\kappa} \hat{t})^{3 / 2}} \quad \text { and thus } \quad \hat{\lambda} \sim(\hat{\kappa} \hat{t})^{1 / 2} .
$$

This is the scaling suggested by our analysis for the cut-off wavenumber for long waves, but we recall that the asymptotics are not valid in this limit. Finally, the most unstable perturbations occur when the two destabilising mechanisms are roughly equal, $\hat{\sigma}_{\text {diff }} \sim \hat{\sigma}_{\text {tran }}$, so

$$
\frac{\hat{\kappa}}{\hat{\lambda}^{2}} \sim \frac{\beta \hat{u}_{0} \hat{\lambda}^{2}}{(\hat{\kappa} \hat{t})^{3 / 2}} \quad \text { and thus } \quad \hat{\lambda} \sim \frac{\hat{\kappa}^{5 / 8} \hat{t}^{3 / 8}}{\beta^{1 / 4} \hat{u}_{0}^{1 / 4}} .
$$

We note that this is not quite the full story: although these scalings allow us to interpret the results of the full stability analysis, they do not predict where in parameter space they can apply. In particular, they give no indication in themselves that a region exists in the SE quadrant where the transverse flow 
term is unimportant (because of the competing thermal and solutal gradients) and so both the cut-off and the favoured wavenumbers are controlled by a balance between diffusion and Saffman-Taylor terms, with the characteristic $t^{-1 / 4}$ scaling emerging as a result.

\section{Summary and conclusions}

We have examined the stability to viscous fingering of a miscible rectilinear displacement front in a porous medium or Hele-Shaw cell, when the viscosity contrast across the front is controlled by two components which diffuse at different rates. Both the growth rates of instabilities and the boundaries of instability change as the front widens and the difference between the lengthscales associated with the two components grows. At early times and for very long waves, the two components interact strongly, so the stabilising influence of one component may compensate for the destabilising influence of another. At later times the front becomes unstable if either component is destabilising, regardless of the other, but the interaction between the two components still controls the growth rates and preferred wavenumber of the instability.

In the long-wave, intermediate-time regime, there is an initially unstable region of parameter space which gradually expands into initially stable regions. This loss of stability as the front diffuses was also remarked upon by Manickam \& Homsy [18] for single-species diffusion with a non-monotonic viscosityconcentration relation. The different roles of the slower- and faster-diffusing components show up in this regime as an asymmetry in the criteria for immediate instability (figure 1), and no simple criterion based solely on the aggregate viscosity profile across the front is able to predict the stability boundary accurately.

In the long-time limit, throughout the unstable region of parameter space we find that the shortest marginally unstable perturbations have wavenumbers that decrease as $t^{-1 / 4}$, in agreement with the single-species case [16]. Throughout most of parameter space the most unstable perturbation occurs at a wavenumber that decreases as $t^{-3 / 8}$; there is, however, a region in which the competing effects of the stabilising and destabilising components produce a favoured wavenumber that decreases as $t^{-1 / 4}$. The difference in scalings is not large but reflects a different balance between the stabilising and destabilising mechanisms acting on an incipient finger. Growing oscillatory perturbations are possible when the faster-diffusing component is destabilising and the slower-diffusing is stabilising, as in the radial geometry considered in [10], but they are always outpaced by non-oscillatory perturbations at lower wavenumber. (In principle it might be possible to observe oscillatory perturbations in a laterally confined channel, so the wavenumbers are restricted as in [10]. In 
this case, it must also be borne in mind that the front will eventually be stabilised as the cut-off wavelength for instability increases beyond the width of the channel.)

The eventual fate of the displacement front is likely to depend both on how the instability is excited and on whether or not fingers are able to grow to reach the nonlinear regime before either the front stabilises or growth rates decline at long times. Nevertheless, these results may provide a useful starting point for the consideration of the dynamics of viscous fingering, and in particular of the tendency for fingering patterns to coarsen over time as the instability mechanism prefers longer and longer disturbances.

Of the three heuristic arguments presented in $\S 1.1$, none entirely captures the fingering process. Argument (i), that if one component promotes fingering then it can occur eventually, is valid for long times. Argument (ii), that it should be the aggregate viscosity contrast that matters, is valid at short times when the more rapidly diffusing component is destabilising, but it fails to recognise the more localised perturbations that can develop when the more slowly diffusing component is destabilising. Argument (iii), that the monotonicity of the viscosity profile is crucial, does not provide a good guide to this problem.

It is also interesting to compare these findings with some previous studies of single-species fingering with a non-monotonic viscosity-concentration relation. The earliest analysis was due to Hickernell \& Yortsos [17], who examined the stability of a step concentration profile in the absence of any dispersive effects: their analysis concluded that instability would occur if there was any segment of decreasing mobility within the mobility profile. This is essentially our heuristic criterion (iii), and as we have seen it does not capture the behaviour of the system investigated here, either at intermediate or at long times.

The subsequent analysis by Manickam \& Homsy [18] incorporated dispersion, but employed a frozen-time approximation. They found that in the long-wave limit the growth rate of perturbations was

$$
\sigma \sim \frac{1}{2} \Lambda m+\mathcal{O}\left(m^{3 / 2}\right), \quad \text { where } \quad \Lambda=-\left(\frac{\left.\frac{\mathrm{d} \mu}{\mathrm{d} c}\right|_{c=0}+\left.\frac{\mathrm{d} \mu}{\mathrm{d} c}\right|_{c=1}}{\frac{\mu(1)}{\mu(0)}+1}\right),
$$

and where the $\mathcal{O}\left(\mathrm{m}^{3 / 2}\right)$ correction term was time-dependent. Thus if $\Lambda>0$ the flow was always unstable at small wavenumbers, and if $\Lambda<0$ the flow was initially stable at small wavenumbers but could later become unstable: numerical simulations suggested that all non-monotonic profiles became unstable eventually, even if $\Lambda<0$. The obvious application of this idea to our problem with the chosen exponential dependence of $\mu$ on $T$ and $C$ yields $\Lambda=\beta_{T}+\beta_{C}$, so in this case it is simply a measure of the aggregate viscosity contrast (our heuristic criterion (ii)); however the long-time numerical findings recall our 
heuristic criterion (iii).

Finally, a heuristic extension of Hickernell \& Yortsos's work by Loggia et al. [22] obtained results that echoed those of [18]; however, the key parameter in their results was an integral expressing the aggregate behaviour of the mobility profile, rather than its limiting behaviour as $c \rightarrow 0$ and $c \rightarrow 1$. In broad terms this is consistent with our findings, in that there is no simple and intuitive criterion that determines the stability; however there is no obvious resemblance between the details of the present analysis and that of [22].

Overall, it appears that the stability properties of double-diffusive miscible displacement are distinct from those of non-monotonic single-species displacement, despite the similarity of the mobility profiles. (We note that our most successful heuristic criterion (i) has no direct equivalent in a single-species displacement.) It would be an interesting exercise to apply the asymptotic approach employed here to the latter problem and to compare the results with the previous studies referred to above.

In conclusion, perhaps the key lesson from the study of this problem is that to predict the occurrence of viscous fingering in a multi-component fluid, it is necessary to take into account not merely how the aggregate properties of the fluid vary across the front, but also how these properties may be subject to differential thermal or molecular diffusion. In more mathematical terms, it also provides a fresh illustration, complementary to those from double-diffusive and reactive-diffusive convection [7,9], of the complexity that is possible when instabilities with different preferred scales occur simultaneously and are intimately linked.

Acknowledgements: This work was initially supported by the NERC/EPSRC Environmental Mathematics and Statistics Programme through a postdoctoral fellowship (ref. NE/B50188X/1) held at the BP Institute, University of Cambridge, and subsequently by the University of Strathclyde under a Faculty of Science Starter Grant (ref. VA5525C). I am grateful to Professor Andrew Woods and Professor Anthony Pearson for helpful discussions of this and related problems during my time at BPI. I am also grateful to two anonymous reviewers for their constructive and instructive comments on an earlier draft.

\section{A Iterative approach to the velocity equation for small $m$}

We wish to solve equation (29), subject to the conditions that $U_{1} \rightarrow 0$ as $\xi \rightarrow \pm \infty$. Unfortunately we cannot write down a closed-form solution to this equation; but intuitively, if $\left|\beta_{C}\right|$ and $\left|\beta_{T}\right|$ are small, so that we can neglect the 
terms proportional to $\beta_{C}$ and $\beta_{T}$ on the LHS of (29), we should be able to develop a series solution in $\beta_{C}$. (This was proposed, although its validity was not confirmed, in [16].)

We set $\beta_{C}=b_{C} \beta$ and $\beta_{T}=b_{T} \beta$, where $\left|b_{C}\right|+\left|b_{T}\right|=1$ and $0<\beta \ll 1$. We then postulate an expansion

$$
U_{1}(\xi)=\frac{2 \beta_{C} m^{2} A(t) \sqrt{t}}{\sqrt{\pi}} \sum_{n=0}^{\infty} \beta^{n} b_{C}^{n} f_{n}(\xi)+\frac{2 \beta_{T} m^{2} B(t) \sqrt{t}}{\sqrt{\pi L e}} \sum_{n=0}^{\infty} \beta^{n} b_{T}^{n} p_{n}(\xi)
$$

and, substituting this into (29), we obtain a sequence of equations:

$$
\begin{array}{r}
\frac{\partial^{2} f_{0}}{\partial \xi^{2}}-4 t m^{2} f_{0}=\mathrm{e}^{-\xi^{2}}, \quad \frac{\partial^{2} p_{0}}{\partial \xi^{2}}-4 t m^{2} p_{0}=\mathrm{e}^{-\xi^{2} / L e} \\
\frac{\partial^{2}}{\partial \xi^{2}}\left\{\begin{array}{c}
f_{n} \\
p_{n}
\end{array}\right\}-4 t m^{2}\left\{\begin{array}{c}
f_{n} \\
p_{n}
\end{array}\right\}=-\left(\frac{b_{C} \mathrm{e}^{-\xi^{2}}}{\sqrt{\pi}}+\frac{b_{T} \mathrm{e}^{-\xi^{2} / L e}}{\sqrt{\pi L e}}\right) \frac{\partial}{\partial \xi}\left\{\begin{array}{c}
f_{n-1} \\
p_{n-1}
\end{array}\right\}
\end{array}
$$

for $n=1,2, \ldots$

Now, the equation

$$
\frac{\partial^{2} f}{\partial \xi^{2}}-4 t m^{2} f=g(\xi)
$$

admits solutions

$$
f(\xi)=\mathcal{F}[g(\xi)] \equiv-\frac{\mathrm{e}^{2 m \sqrt{t} \xi}}{4 m \sqrt{t}} \int_{\xi}^{\infty} \mathrm{e}^{-2 m \sqrt{t} x} g(x) \mathrm{d} x-\frac{\mathrm{e}^{-2 m \sqrt{t} \xi}}{4 m \sqrt{t}} \int_{-\infty}^{\xi} \mathrm{e}^{2 m \sqrt{t} x} g(x) \mathrm{d} x,
$$

and as long as $g(\xi)$ is well-behaved as $\xi \rightarrow \pm \infty$, this solution satisfies the boundary conditions $f(\xi) \rightarrow 0$ as $\xi \rightarrow \pm \infty$. We therefore have

$$
f_{0}(\xi)=\mathcal{F}\left[\mathrm{e}^{-\xi^{2}}\right] \quad \text { and } \quad f_{n}(\xi)=\mathcal{F}\left[-\left(\frac{b_{C} \mathrm{e}^{-\xi^{2}}}{\sqrt{\pi}}+\frac{b_{T} \mathrm{e}^{-\xi^{2} / L e}}{\sqrt{\pi L e}}\right) \frac{\partial f_{n-1}}{\partial \xi}\right]
$$

for $n=1,2, \ldots$, and

$$
p_{0}(\xi)=\mathcal{F}\left[\mathrm{e}^{-\xi^{2} / L e}\right] \quad \text { and } \quad p_{n}(\xi)=\mathcal{F}\left[-\left(\frac{b_{C} \mathrm{e}^{-\xi^{2}}}{\sqrt{\pi}}+\frac{b_{T} \mathrm{e}^{-\xi^{2} / L e}}{\sqrt{\pi L e}}\right) \frac{\partial p_{n-1}}{\partial \xi}\right]
$$

for $n=1,2, \ldots$

If $g(\xi)$ is odd / even then $\mathcal{F}[g(\xi)]$ is also odd / even respectively; this means that $U_{1 n}(\xi)$ is even / odd when $n$ is even / odd respectively. This fact is relevant because when we take the inner product $\left\langle\mathrm{e}^{-\xi^{2}} U_{1}\right\rangle_{C}$, only the even terms in the series will contribute anything. 
We also have

$$
\frac{\partial}{\partial \xi} \mathcal{F}[g(\xi)]=-\frac{1}{2} \mathrm{e}^{2 m \sqrt{t} \xi} \int_{\xi}^{\infty} \mathrm{e}^{-2 m \sqrt{t} x} g(x) \mathrm{d} x+\frac{1}{2} \mathrm{e}^{-2 m \sqrt{t} \xi} \int_{-\infty}^{\xi} \mathrm{e}^{2 m \sqrt{t} x} g(x) \mathrm{d} x .
$$

Consider the sequences of functions defined by

$$
\left\{\begin{array}{l}
g_{n+1}(\xi) \\
q_{n+1}(\xi)
\end{array}\right\}=-\left(\frac{b_{C} \mathrm{e}^{-\xi^{2}}}{\sqrt{\pi}}+\frac{b_{T} \mathrm{e}^{-\xi^{2} / L e}}{\sqrt{\pi L e}}\right) \frac{\partial}{\partial \xi}\left\{\begin{array}{l}
\mathcal{F}\left[g_{n}(\xi)\right] \\
\mathcal{F}\left[q_{n}(\xi)\right]
\end{array}\right\}
$$

with $g_{0}(\xi)=\mathrm{e}^{-\xi^{2}}$ and $q_{0}(\xi)=\mathrm{e}^{-\xi^{2} / L e}$, so that $f_{n}(\xi)=\mathcal{F}\left[g_{n}(\xi)\right]$ and $p_{n}(\xi)=$ $\mathcal{F}\left[q_{n}(\xi)\right]$. We wish to show that these sequences are convergent, and to do so we will proceed iteratively. We will obtain a convergence criterion for $g_{n}(\xi)$; since the recurrence relations for $g_{n}$ and $p_{n}$ are identical, this will also be the convergence criterion for $p_{n}$.

Suppose that $\left|g_{n}(\xi)\right| \leq C_{n} \mathrm{e}^{-\xi^{2}}+D_{n} \mathrm{e}^{-\xi^{2} / L e}$ for some constants $C_{n} \geq 0$ and $D_{n} \geq 0$, and for convenience define $M=m \sqrt{t}$. We then have

$$
\begin{aligned}
\left|g_{n+1}(\xi)\right| \leq & \left(\frac{b_{C} \mathrm{e}^{-\xi^{2}}}{\sqrt{\pi}}+\frac{b_{T} \mathrm{e}^{-\xi^{2} / L e}}{\sqrt{\pi L e}}\right) \frac{1}{2} \int_{-\infty}^{\infty} \mathrm{e}^{-2 M|x-\xi|}\left|g_{n}(x)\right| \mathrm{d} x \\
\leq & C_{n}\left(\frac{b_{C} \mathrm{e}^{-\xi^{2}}}{\sqrt{\pi}}+\frac{b_{T} \mathrm{e}^{-\xi^{2} / L e}}{\sqrt{\pi L e}}\right) \frac{1}{2} \int_{-\infty}^{\infty} \mathrm{e}^{-2 M|x-\xi|} \mathrm{e}^{-x^{2}} \mathrm{~d} x \\
& +D_{n}\left(\frac{b_{C} \mathrm{e}^{-\xi^{2}}}{\sqrt{\pi}}+\frac{b_{T} \mathrm{e}^{-\xi^{2} / L e}}{\sqrt{\pi L e}}\right) \frac{1}{2} \int_{-\infty}^{\infty} \mathrm{e}^{-2 M|x-\xi|} \mathrm{e}^{-x^{2} / L e} \mathrm{~d} x \\
= & C_{n}\left(\frac{b_{C} \mathrm{e}^{-\xi^{2}}}{\sqrt{\pi}}+\frac{b_{T} \mathrm{e}^{-\xi^{2} / L e}}{\sqrt{\pi L e}}\right) h(\xi, M) \\
& +D_{n}\left(\frac{b_{C} \mathrm{e}^{-\xi^{2}}}{\sqrt{\pi}}+\frac{b_{T} \mathrm{e}^{-\xi^{2} / L e}}{\sqrt{\pi L e}}\right) \sqrt{L e} h\left(\frac{\xi}{\sqrt{L e}}, M \sqrt{L e}\right)
\end{aligned}
$$

where $h(\xi, M)=\frac{\sqrt{\pi} \mathrm{e}^{M^{2}}}{4}\left[\mathrm{e}^{2 M \xi}(1-\operatorname{erf}(M+\xi))+\mathrm{e}^{-2 M \xi}(\operatorname{erf}(M-\xi)-1)\right]$.

The maximum of $h(\xi, M)$ over $\xi$ occurs at $\xi=0$, and the maximum of $h(0, M)$ over $M$ occurs at $M=0$; we conclude that

$$
\begin{aligned}
& \left|g_{n+1}(\xi)\right| \leq\left[C_{n}\left(\frac{b_{C} \mathrm{e}^{-\xi^{2}}}{\sqrt{\pi}}+\frac{b_{T} \mathrm{e}^{-\xi^{2} / L e}}{\sqrt{\pi L e}}\right)+D_{n} \sqrt{L e}\left(\frac{b_{C} \mathrm{e}^{-\xi^{2}}}{\sqrt{\pi}}+\frac{b_{T} \mathrm{e}^{-\xi^{2} / L e}}{\sqrt{\pi L e}}\right)\right] h(0,0) \\
& =\left[C_{n}\left(\frac{b_{C} \mathrm{e}^{-\xi^{2}}}{2}+\frac{b_{T} \mathrm{e}^{-\xi^{2} / L e}}{2 \sqrt{L e}}\right)+D_{n} \sqrt{L e}\left(\frac{b_{C} \mathrm{e}^{-\xi^{2}}}{2}+\frac{b_{T} \mathrm{e}^{-\xi^{2} / L e}}{2 \sqrt{L e}}\right)\right] .
\end{aligned}
$$


We can therefore define the upper bounds at the next iteration as

$$
\left[\begin{array}{c}
C_{n+1} \\
D_{n+1}
\end{array}\right]=\left[\begin{array}{cc}
\frac{b_{C}}{2} & \frac{b_{C} \sqrt{L e}}{2} \\
\frac{b_{T}}{2 \sqrt{L e}} & \frac{b_{T}}{2}
\end{array}\right] \cdot\left[\begin{array}{c}
C_{n} \\
D_{n}
\end{array}\right] .
$$

The eigenvalues of the matrix are $\lambda=0$ and $\lambda=\left(b_{C}+b_{T}\right) / 2$. Thus, substituting our upper bound into (A.1), we find that

$$
\begin{aligned}
\left|U_{1}(\xi)\right| \leq & \frac{2 m^{2}\left|\beta_{C} A(t)\right| \sqrt{t}}{\sqrt{\pi}} \sum_{n=0}^{\infty} \beta^{n}\left|b_{c}\right|^{n}\left|f_{n}(\xi)\right| \\
& +\frac{2 m^{2}\left|\beta_{T} B(t)\right| \sqrt{t}}{\sqrt{\pi L e}} \sum_{n=0}^{\infty} \beta^{n}\left|b_{T}\right|^{n}\left|p_{n}(\xi)\right| \\
\leq & \frac{2 m^{2}\left|\beta_{C} A(t)\right| \sqrt{t}}{\sqrt{\pi}} \sum_{n=0}^{\infty}\left|\frac{\beta b_{C}\left(b_{C}+b_{T}\right)}{2}\right|^{n}\left|f_{0}(\xi)\right| \\
& +\frac{2 m^{2}\left|\beta_{T} B(t)\right| \sqrt{t}}{\sqrt{\pi L e}} \sum_{n=0}^{\infty}\left|\frac{\beta b_{T}\left(b_{C}+b_{T}\right)}{2}\right|^{n}\left|p_{0}(\xi)\right| .
\end{aligned}
$$

We conclude that the series converges if

$$
\left|\frac{\beta b_{C}\left(b_{C}+b_{T}\right)}{2}\right| \leq\left|\frac{\beta_{C}}{2}\right|<1 \quad \text { and } \quad\left|\frac{\beta b_{T}\left(b_{C}+b_{T}\right)}{2}\right| \leq\left|\frac{\beta_{T}}{2}\right|<1
$$

where we have used the condition that $\left|b_{C}\right|+\left|b_{T}\right|=1$.

Finally, noting that when we calculate $\left\langle\mathrm{e}^{-\xi^{2}} U_{1}\right\rangle_{C}$ and $\left\langle\mathrm{e}^{-\xi^{2} / L e} U_{1}\right\rangle_{T}$ the oddnumbered terms in the series vanish, we conclude that when $\beta_{C}$ and $\beta_{T}$ satisfy the convergence conditions (A.17), we have

$$
\begin{aligned}
\left\langle\mathrm{e}^{-\xi^{2}} U_{1}(\xi)\right\rangle_{C}= & \frac{2 \beta_{C} m^{2} A \sqrt{t}}{\sqrt{\pi}}\left\langle\mathrm{e}^{-\xi^{2}} f_{0}(\xi)\right\rangle_{C} \\
& +\frac{2 \beta_{T} m^{2} B \sqrt{t}}{\sqrt{\pi L e}}\left\langle\mathrm{e}^{-\xi^{2}} p_{0}(\xi)\right\rangle_{C}+\mathcal{O}\left(\left(\left|\beta_{C}\right|+\left|\beta_{T}\right|\right)^{3}\right), \\
\left\langle\mathrm{e}^{-\xi^{2} / L e} U_{1}(\xi)\right\rangle_{T}= & \frac{2 \beta_{C} m^{2} A \sqrt{t}}{\sqrt{\pi}}\left\langle\mathrm{e}^{-\xi^{2} / L e} f_{0}(\xi)\right\rangle_{T} \\
& +\frac{2 \beta_{T} m^{2} B \sqrt{t}}{\sqrt{\pi L e}}\left\langle\mathrm{e}^{-\xi^{2} / L e} p_{0}(\xi)\right\rangle_{T}+\mathcal{O}\left(\left(\left|\beta_{C}\right|+\left|\beta_{T}\right|\right)^{3}\right) .
\end{aligned}
$$

In other words, for sufficiently small values of $\left|\beta_{C}\right|+\left|\beta_{T}\right|$, the solution that we obtain by neglecting the first derivatives of $U_{1}$ on the LHS of (29) is accurate to second order in $\left(\left|\beta_{C}\right|+\left|\beta_{T}\right|\right)$. 


\section{References}

[1] G. M. Homsy, Viscous fingering in porous media, Annual Review of Fluid Mechanics 19 (1987) 271-311.

[2] P. G. Saffman, G. I. Taylor, The penetration of a fluid into a porous medium or Hele-Shaw cell containing a more viscous liquid, Proceedings of the Royal Society A 245 (1242) (1958) 312-329.

[3] L. P. Dake, Fundamentals of reservoir engineering, Vol. 8 of Developments in Petroleum Science, Elsevier, 1978.

[4] R. B. Needham, P. H. Doe, Polymer flooding review, Journal of Petroleum Technology (1987) 1503-1507(SPE paper 17140).

[5] CRC Press, CRC Handbook of Chemistry and Physics, 82nd Edition (2001).

[6] V. Stefánsson, Geothermal reinjection experience, Geothermics 26 (1997) 99139.

[7] J. S. Turner, Multicomponent convection, Annual Review of Fluid Mechanics 17 (1985) 11-44.

[8] A. De Wit, Fingering of chemical fronts in porous media, Physical Review Letters 87 (5) (2001) 87.054502.

[9] J. D'Hernoncourt, J. H. Merkin, A. De Wit, Front fingering and complex dynamics driven by the interaction of buoyancy and diffusive instabilities, Physical Review E 76 (2007) 035301(R).

[10] D. Pritchard, The instability of thermal and fluid fronts during radial injection in a porous medium, Journal of Fluid Mechanics 508 (2004) 133-163.

[11] O. M. Phillips, Flow and Reactions in Permeable Rocks, Cambridge University Press, 1991.

[12] E. Lajeunesse, J. Martin, N. Rakotomalala, D. Salin, Y. C. Yortsos, Miscible displacement in a Hele-Shaw cell at high rates, Journal of Fluid Mechanics 398 (1999) 299-319.

[13] D. Schafroth, N. Goyal, E. Meiburg, Miscible displacements in Hele-Shaw cells: nonmonotonic viscosity profiles, European Journal of Mechanics B 26 (2007) 444-453.

[14] C. T. Tan, G. M. Homsy, Stability of miscible displacements in porous media: radial source flow, Physics of Fluids 30 (5) (1987) 1239-1245.

[15] C. T. Tan, G. M. Homsy, Stability of miscible displacements in porous media: rectilinear flow, Physics of Fluids 29 (11) (1986) 3549-3556.

[16] Y. Ben, E. A. Demekhin, H.-C. Chang, A spectral theory for small-amplitude miscible fingering, Physics of Fluids 14 (3) (2002) 999-1010. 
[17] F. J. Hickernell, Y. C. Yortsos, Linear stability of miscible displacement processes in porous media in the absence of dispersion, Studies in Applied Mathematics 74 (1986) 93-115.

[18] O. Manickam, G. M. Homsy, Stability of miscible displacements in porous media with nonmonotonic viscosity profiles, Physics of Fluids A 5 (6) (1993) 13561367.

[19] G. Barenblatt, Scaling, Self-Similarity and Intermediate Asymptotics, Cambridge University Press, 1996.

[20] K. K. Tan, R. B. Thorpe, The onset of convection driven by buoyancy effects caused by various modes of transient heat conduction: Part I. Transient Rayleigh numbers, Chemical Engineering Science 54 (1999) 225-238.

[21] A. Riaz, M. Hesse, H. A. Tchelepi, F. M. Orr, Onset of convection in a gravitationally unstable diffusive boundary layer in porous media, Journal of Fluid Mechanics 548 (2006) 87-111.

[22] D. Loggia, D. Salin, Y. C. Yortsos, The effect of dispersion on the stability of non-monotonic mobility profiles in porous media, Physics of Fluids 10 (3) (1998) 747-749.

[23] S. S. S. Cardoso, A. W. Woods, The formation of drops through viscous instability, Journal of Fluid Mechanics 289 (1995) 351-378. 